\title{
The Hidden Group Structure of Quantum Groups: Strong Duality, Rigidity and Preferred Deformations
}

\author{
P. Bonneau ${ }^{1}$, M. Flato ${ }^{1}$, M. Gerstenhaber ${ }^{2}$, G. Pinczon ${ }^{1}$ \\ ${ }^{1}$ Université de Bourgogne, Laboratoire de Physique Mathématique, B.P. 138, 21004 Dijon \\ Cedex - France. e-mail: flato@satie.u-bourgogne.fr \\ 2 Department of Mathematics, University of Pennsylvania, Philadelphia, PA 19104-6395, U.S.A. \\ e-mail: mgersten@mail.sas upenn.edu and murray@math.upenn.edu
}

Received:14 June 1993

\begin{abstract}
A notion of well-behaved Hopf algebra is introduced; reflexivity (for strong duality) between Hopf algebras of Drinfeld-type and their duals, algebras of coefficients of compact semi-simple groups, is proved. A hidden classical group structure is clearly indicated for all generic models of quantum groups. Moyalproduct-like deformations are naturally found for all FRT-models on coefficients and $C^{\infty}$-functions. Strong rigidity $\left(H_{b i}^{2}=\{0\}\right)$ under deformations in the category of bialgebras is proved and consequences are deduced.
\end{abstract}

\section{Introduction}

There presently exist at least four models of quantum groups, introduced respectively by Drinfeld (D-model) [6], Jimbo (J-model) [16], Faddeev-ReshetikhinTakhtajan (FRT-model) [9] and Woronowicz (W-model) [23]. We apologize for missing other models or authors. All these models are Hopf algebras that intend to be "deformations," in the following sense: they depend on a parameter, say $q$ (or $e^{i t}$ ), and when $q=1$ (or $t=0$ ), one finds a very classical and well known Hopf algebra, such as, e.g.: the enveloping algebra of a simple Lie algebra (D), the algebra of coefficients on an algebraic reductive group (FRT), an algebra of continuous functions on a compact group (W). It is often claimed that the classical limit of the $\mathrm{J}$-model is the enveloping algebra of the corresponding simple Lie algebra; however this claim is not quite correct (see e.g. [5] or [3]): in fact, the classical limit of the J-model is an extension of $\mathscr{U}(g)$ by $r$ parities $(r=\operatorname{rank} g)$. As a matter of fact this should have been obvious even a priori, since all deformations of the (multiplicative structure of the) enveloping algebra of a simple algebra are trivial [8], while the J-model is a non-trivial deformation of its classical limit (see the end of this introduction). It is also often asserted that the D and FRT-models are mutually dual. Although this claim seems quite reasonable because generators of D-models can be found in the dual of FRT-models [9], a canonical duality $*$ such that $\mathrm{D}^{*}=\mathrm{FRT}$ and $\mathrm{FRT}^{*}=\mathrm{D}$ has not yet been constructed.

Here is a short summary of some puzzling problems concerning quantum groups: 
a) A first problem is the meaning that one should give to the word "deformation." A very good discussion is given in [11]. If we take the usual notion of the third author, then the D-model is a deformation of $\mathscr{U}(g)$; Drinfeld has shown that this deformation is trivial from the algebra viewpoint, so that the only thing which is really deformed is the coproduct. We shall discuss later the J-model. For the FRT model, [11] shows that defining relations of the type $\mathscr{R} T_{1} T_{2}=T_{2} T_{1} \mathscr{R}$ do not necessarily define a deformation, even if $\mathscr{R}$ is Yang-Baxter; however, one shows [11] that the FRT-model is a deformation if $G=S U(n)$. The problem of showing that the FRT-model is a deformation for other $G$, such as $S O(n), S p(n)$, has not been solved up to now.

b) A second problem is related to duality. It is not only a problem concerning quantum groups, but really a problem concerning Hopf algebras in general, when not finite dimensional. For such a Hopf algebra $A$, the algebraic dual $A^{\prime}$ is not a Hopf algebra, and $A \subset{ }_{\neq} A^{\prime \prime}$.

Therefore if one starts with the naive, but suggestive, idea saying that if the D-model is a deformation then the FRT-model, being its dual, should also be a deformation, one gets stuck in trying to prove it rigorously. What is necessary, as noted in [9] (Remark 23), is a theory of reflexive Hopf algebras which avoids the technical difficulties coming from the lack of reflexivity of the usual algebraic theory. Obviously, such a theory has to be a topological one!

c) A third problem is to give an interpretation of the Tannaka-Krein philosophy in the case of quantum groups: it has often been noticed that, in the generic case, finite dimensional representations of a quantum group are (essentially) representations of its classical limit. So the algebras involved should be the same, and this is justified by the above-mentioned rigidity result of Drinfeld. Such a remark shows that the initial classical group is still there, acting as some "hidden variables" of this quantum group theory, and it is an interesting challenge to discover these hidden variables.

d) Related to the third problem is the result of the third author and S.D. Schack on preferred presentations [12]. Assuming that some duality does exist for which the FRT-model is dual to the D-model, it will follow that the FRT-model is a deformation of the algebra of coefficients of $G$. As the algebra structure of the D-model remains the initial one, the coalgebra structure of the FRT-model remains also the initial one, so one should be able to realize the FRT-model as a deformation of the algebra of coefficients of $G$, with unchanged coproduct (a preferred deformation).

e) Finally (once more if a convenient duality does exist) the FRT-model should extend to $C^{\infty}$-functions and provide a deformation of the usual product with the Poisson bracket as leading term. This picture looks very much like the Moyal product of quantum mechanics, and is given heuristically in [6] as a justification for the name quantum groups (see also [1]).

The goal of the present paper is to give an answer to the above questions. We shall:

i) recover FRT-models from D-models by a suitable duality argument.

ii) show that there exists a preferred deformation (in the Gerstenhaber-Schack sense) of the Hopf algebra of coefficient functions on $G$ ( $G$ a compact connected Lie group) satisfying the FRT-relation $\mathscr{R} T_{1} T_{2}=T_{2} T_{1} \mathscr{R}$, with $\mathscr{R}$ Yang-Baxter as in [9], and that this deformation extends to a deformation of the algebra $C^{\infty}(G)$. 
iii) discuss properties of D-models, FRT-models and J-models in the framework of deformation theory.

In order to get these results, we introduce several new concepts, such as those of well-behaved Hopf algebras and deformation theory of topological algebras, which are of interest by themselves; for instance, the (strong) dual of a well-behaved Hopf algebra is a well behaved Hopf algebra, and the (topological) deformation theory of a well-behaved Hopf algebra is equivalent to the (topological) deformation theory of its (strong) dual. Let us give a brief survey of the principal results of the paper.

First, we introduce a category of topological Hopf algebras for which duality works as if the algebra was finite dimensional. For this reason, we call them "well-behaved" Hopf algebras. If $A$ is such an algebra, then its (strong) dual $A^{*}$ is also a well-behaved Hopf algebra, with Hopf structure obtained by transposition of the Hopf structure of $A$, and $A^{* *}=A$ as Hopf algebras. We show that any countable-dimension Hopf algebra can be given a natural topology for which it is a well-behaved Hopf algebra. Moreover any $H(G)=C^{\infty}(G), G$ a compact connected Lie group, is a well-behaved Hopf algebra, and so is its dual $A(G)$, the convolution algebra of distributions on $G$. There are many other examples, essentially all Hopf algebras of interest.

While FRT-models are usually constructed for complex reductive algebraic groups, this context can be replaced by that of compact connected Lie groups. We prefer to deal with the second case, since this brings us back to the usual harmonic analysis on (compact) Lie groups, and also because we believe in the power and simplicity of Weyl's unitary trick. In Sect. 2, related to the Tanaka-Krein philosophy, we give a complete description of the well-behaved Hopf algebra $\mathscr{H}(G)$ ( $G$ a compact connected Lie group) of coefficients of $G$, and of its dual $\mathscr{A}(G)$; our description is in fact an algebraic version of the Fourier transform on compact groups.

In the third section, since the algebras to be deformed are topological, we have to present a topological version of the Gerstenhaber theory of deformations. This construction is parallel to the usual one (needed technical results are given in Appendix 3). The main result is that to deform (in the topological sense) a wellbehaved Hopf algebra is tantamount to deforming its strong dual. Note, however, that in the case of countable dimension Hopf algebras, the purely algebraic deformation theory and topological deformation theory do coincide.

In the fourth section, we study deformations of the well-behaved algebras $\mathscr{H}(G), H(G)$, and their duals $\mathscr{A}(G), A(G)$, previously introduced. Just like Drinfeld's result for $\mathscr{U}(g)$, we show that $A(G)$ and $\mathscr{A}(G)$ are rigid in the associative category, and the new coproduct is obtained from the initial one by a twist, so it is still quasi-coassociative and quasi-cocommutative (see [2] for a rigidity interpretation of this result, and a discussion of cohomology and deformations of quasicoassociative bialgebras). It should nevertheless be remarked at this point that one of the basic results of the present paper (generalizing a similar result of strong rigidity for the case of $S U(2)$ [3] to the general case of a compact connected Lie group) is as follows: while the J-model (as will be explained later) does not exhibit any rigidity, and the D-model, as shown by Drinfeld is rigid under global deformations, what we show for $A(G)$ and $\mathscr{A}(G)$ is strong rigidity. That is, they are rigid under both infinitesimal and a fortiori global deformations in the category of bialgebras because the corresponding second cohomology space, as defined in [3], vanishes identically. 
We thus have a kind of Whitehead lemma for our version of quantum groups. By duality, any coassociative deformation of $\mathscr{H}(G)$, or $H(G)$, has a preferred presentation. For $\mathscr{H}(G)$, this corresponds to the Gerstenhaber-Schack result, while for $H(G)$ it is new. Moreover, we show that in such a deformation of $\mathscr{H}(G)$, or $H(G)$, the product of central functions (e.g., characters) is unchanged, something which was never noticed. Finally, we show that preferred deformations of $H(G)$ do restrict to $\mathscr{H}(G)$.

In the fifth section, we introduce a notion of quotient deformation, by showing that preferred deformations of $\mathscr{H}(G)$, or $H(G)$, do produce preferred deformations of $\mathscr{H}(G / \Gamma)$, or $H(G / \Gamma)$, if $\Gamma$ is a closed normal subgroup of $G$. This result is interesting even if $G$ is simple (it can be used e.g. for $G=S U(2)$ and $G / \Gamma=S O(3)$ ). This extends naturally to cases where $\Gamma$ is closed but not necessarily normal. There one can still define $\mathscr{H}(G / \Gamma)$, respectively $H(G / \Gamma)$, which now, however, are comodule algebras over $\mathscr{H}(G)$, respectively $H(G)$. For a comodule algebra over a bialgebra the notion of a preferred deformation (relative to a preferred deformation of the bialgebra) is still defined: we require that the bialgebra and the comodule coalgebra structure over it simultaneously so deform that we continue to have a comodule algebra over the bialgebra, with the comultiplications of both the bialgebra and comodule algebra remaining unchanged. In this way it is meaningful to speak, in particular, of preferred deformations of homogeneous spaces.

In Sect. 6, we achieve another important goal of this paper: we justify duality between D-models and FRT-models. Here is a brief sketch: Denote by $\mathscr{U}_{t}$ the D-model deformation of $\mathscr{U}(g)$. We make a "good choice" of a Drinfeld isomorphism $\varphi: \mathscr{U}_{t} \simeq \mathscr{U}(g)[[t]]$. Using $\varphi$, we imbed $\mathscr{U}_{t}$ as a subalgebra of $\mathscr{A}(G)[[t]]$ or $A(G)[[t]]$ ( $G$ a compact connected semi-simple Lie group with complexified Lie algebra $g$ ). We then show that the coproduct $\tilde{\Delta}$ of $\mathscr{U}_{t}$ extends to $\mathscr{A}(G)[[t]]$ (or $A(G)[[t]])$, and so does the antipode and counit. Therefore we get Hopf deformations of topological Hopf algebras $\mathscr{A}(G)[[t]]$ and $A(G)[[t]]$, and (using the main result of Sect. 3) preferred Hopf deformations of $\mathscr{H}(G)$ and $H(G)$, which satisfy the FRT-relation $\mathscr{R} T_{1} T_{2}=T_{2} T_{1} \mathscr{R}$ with $\mathscr{R}$ Yang-Baxter. Let us emphasize that this works not only for $G=S U(n), S O(n)$ or $S p(n)$, as in [9], but also for $G=\operatorname{Spin}(n)$, and for exceptional $G$. For instance, in the case of $\operatorname{Spin}(2 p)$, it predicts the existence of a preferred deformation of type $\mathscr{R} T_{1} T_{2}=T_{2} T_{1} \mathscr{R}, \mathscr{R}$ Yang-Baxter, based on the direct sum of the spinor-representations (see also Remark 23 in [9]); such a model has never been explicitly described up to now. Note that our result of realization of quantum groups as preferred deformations of $\mathscr{H}(G)$ and $H(G)$ (instead of algebras defined by generators and relations) gives a complete answer to a question of [11] (solved for $S U(n)$ in [11]). On the other hand, the above-mentioned "good choice" of the Drinfeld isomorphism $\varphi$ is far from being explicit; on the contrary, it seems to be an incredibly complicated problem to give an explicit $\varphi$. This can be said as follows: hidden variables (actually the initial group $G$ ) do exist for quantum groups, but it is a non-trivial problem to give an explicit realization!

In Sect. 7, we discuss the $\mathrm{J}$ model, in the case $g=s l(2), G=S U(2)$. It is usually thought that the J-model is a clever, but equivalent, notation for the D-model. We show that, though clever, it is not at all equivalent. Denote by $A_{t}$ the J-model and $\tilde{A}_{t}$ the same with $t$ a formal parameter. It is an easy matter to specialize (or "contract") $A_{t}$ to $t=0$. The $A_{0}$ obtained is not $\mathscr{U}(g)$, but an extension of $\mathscr{U}(g)$ by a parity $C\left(C^{2}=1\right)$. This constitutes a first difference. So $A_{0}$ is not a domain. Since $\tilde{A}_{t}$ is a domain, $\tilde{A}_{t}$ is a non-trivial deformation of $A_{0}$, a second difference with the D-model. Moreover, we can consider $\tilde{A}_{t_{0}+t}$ as a deformation of $A_{t_{0}}$, and we show 
that it is a non-trivial one, a third difference. We have shown in [3] that $A_{t}$, for generic $t$, can be realized as a dense subalgebra of $\mathscr{A}(G)$. Actually, $\mathscr{A}(G)$ has several nice properties: it is rigid as an algebra, the D-model can be recovered as a dense subalgebra of deformation of the Hopf algebra $\mathscr{A}(G)$, duality provides the FRT model (since $\mathscr{A}(G)^{*}=\mathscr{H}(G)$ ), finite dimensional representations of $\mathscr{A}(G)$ and $G$ are the same, etc. So, in our opinion, $\mathscr{A}(G)$ is exactly the model of hidden variables of quantum groups, in that case.

The paper contains four appendices, which introduce needed technical material and present the terminology and notations we use. In particular, Appendix 2 is devoted to the natural $\mathscr{L} \mathscr{F}$-nuclear topology on countable dimension spaces, a very simple, but fundamental notion, since it provides any countable dimension Hopf algebra with a well-behaved Hopf structure.

Many ideas of the present paper originate in [3], where the case $G=S U(2)$ is explicitly treated.

Finally, any reader will notice that this paper is a discussion of the generic case of quantum groups. We do not discuss the "roots of unity" case, because we are dealing with deformation theory. Some explicit deformation formulas, e.g., the Moyal product remain well-defined when $\hbar$ is a root of unity, but the latter seems never to have been discussed. Nevertheless we believe that the really interesting applications of quantum groups should come from the root of unity case, a case that should be compared with representation theory on finite fields rather than with real (or complex) Lie group theory.

\section{Well-Behaved Topological Bialgebras}

We refer to Appendix 4 for notions of topological algebras, bialgebras, etc..

(1.1) The Framework. Let us assume that $A$ is a topological bialgebra, and moreover, that as a complete topological vector space (c.t.v.s.) $A$ is nuclear, and Fréchet or dual of Fréchet. Then $A$ is Montel [21], so, by (A.1.3), $A$ is reflexive. Using (A.1.5), transposition of the product and coproduct of $A$ defines a coproduct and a product on $A^{*}$, so $A^{*}$ becomes a topological bialgebra. Now $A^{*}$, as a t.v.s., satisfies exactly the same conditions as $A$, so we can repeat the transposition operation, and since $A$ is reflexive, we recover the initial bialgebra structure of $A$.

If we assume that $A$ is associative (with unit), then $A^{*}$ will be coassociative (with counit), and if we assume that $A$ is a topological Hopf algebra, then the counit of $A$ will define the unit of $A^{*}$; the counit of $A^{*}$ is the evaluation on the unit of $A$, and the antipode of $A^{*}$ is the transpose of the antipode of $A$, so $A^{*}$ is also a topological Hopf algebra. Therefore, we introduce the following definition:

(1.2) Definition. A topological algebra (resp: bialgebra, Hopf algebra) is wellbehaved if (as a c.t.v.s.) it is nuclear and Fréchet, or nuclear and dual of Fréchet.

Now, we summarize the results of this section:

(1.3) Proposition. When $A$ is a well-behaved topological bialgebra (resp: Hopf algebra) then the transposition defines on $A^{*}$ a well-behaved topological bialgebra (resp: Hopf algebra) structure, and the initial structure of $A=A^{* *}$ is recovered by transposition of the structure of $A^{*}$. 
(1.4) Remarks. The classical algebraic theory of Hopf algebras suffers from an obvious lack of reflexivity, as soon as the algebra is not finite dimensional, and (1.3) shows that the category of well-behaved algebras is really of interest, because reflexivity now holds and everything works almost as in the finite dimensional case. While the condition of being well-behaved may seem unnatural, in the rest of this section, we show that almost all bialgebras or Hopf algebras of interest are in fact well-behaved.

(1.5) Natural Topology. First, we note that bialgebras or Hopf algebras used in algebraic theories always have countable dimension. In this case, Appendix 2 provides a natural topology on $A$, and the following result shows that it is the good one:

(1.5.1) Proposition. If $A$ is a countable dimension bialgebra (resp: Hopf algebra), and if $A$ is given its natural topology (see A2), then $A$ is a well-behaved topological bialgebra (resp: Hopf algebra).

Proof. By (A.2.4), the natural topology is nuclear and complete, and $A$ is reflexive. By (A.2.5), $A^{*}$ is Fréchet, so we have only to prove that $A$ is a topological bialgebra (resp: Hopf algebra), but it is an obvious consequence of (A.2.8) and (A.2.2).

Q.E.D.

(1.6) Example. Let $\mathbb{C}[t]$ be the space of polynomials, with its natural topology, and $\mathbb{C}[[t]]$ its dual (see A.2.10)). We define a well-behaved topological Hopf algebra structure on $\mathbb{C}[t]$ by: $t^{n} \times t^{p}=\left(\begin{array}{c}n+p \\ p\end{array}\right) t^{n+p}$, coproduct $\delta\left(t^{n}\right)=$ $\sum_{i=0}^{n} t^{i} \otimes t^{n-i}$, counit $\varepsilon\left(t^{n}\right)=\delta_{n 0}$, antipode $S\left(t^{n}\right)=(-1)^{n} t^{n}$. Using (1.3), we get a well-behaved topological Hopf algebra structure on $\mathbb{C}[[t]]$.

It is easily seen that the product is the usual product of $\mathbb{C}[[t]]$, and the coproduct is given by:

$$
f(t) \in \mathbb{C}[[t]], \quad \Delta(f)=f\left(t+t^{\prime}\right) \in \mathbb{C}\left[\left[t, t^{\prime}\right]\right] \simeq \mathbb{C}[[t]] \hat{\otimes} \mathbb{C}[[t]] .
$$

(1.7) The algebras $H$ and $A$. Let $G$ be a compact connected Lie group, and $H(G)=C^{\infty}(G)$. Here the topology of $H$ is the usual Fréchet topology, which is nuclear [13]. Now, the product on $H$ is the pointwise abelian product of functions, the coproduct is defined by $\delta(f)(x, y)=f(x y)$, using the standard isomorphism $H(G) \hat{\otimes} H(G) \simeq H(G \times G)$, the counit is the Dirac distribution at the unity of $G$, and the antipode is $S(f)(x)=f\left(x^{-1}\right)$, so we get a well-behaved topological Hopf algebra. Using (1.3), $H(G)^{*}=A(G)$ is also a well-behaved topological Hopf algebra, but $H(G)$ * is the space of distributions on $G$, and it is easy to check that the product so obtained is the usual convolution product of distributions; identifying $G$ and Dirac distributions, one gets from the compactness of $G$ a topological inclusion $G \subset A(G)$. Since $G^{\perp}=\{0\}, \overline{\operatorname{Vect}(G)}=A(G)$, where $\operatorname{Vect}(G)$ is the linear span of $G$. Then, denoting by $\Delta$ the transpose of the product of $H(G)$, one has $\Delta(x)=x \otimes x, x \in G$. Finally, $1_{A(G)}=1_{G}$, and the counit of $A(G)$ is the trivial representation of $G$. Summarizing:

(1.7.1) Proposition. $H(G)$ and $A(G)$ are well-behaved topological Hopf algebras. The product on $A(G)$ is the convolution product of distributions, the coproduct is $\Delta(x)=x \otimes x, x \in G$, and the counit is the trivial representation of $G$. 


\section{The Hopf Algebra of Coefficients of $G$, and its Dual}

Let $G$ be a compact connected Lie group and $g$ be the complexification of the Lie algebra of $G$. We have already introduced in (1.7) the well-behaved topological Hopf algebras $H=H(G)=C^{\infty}(G)$, and its dual $A=A(G)$, the well-behaved Hopf algebra of distributions. We shall now present another model of well-behaved topological Hopf algebra naturally associated to $G$ : the algebra of coefficients $\mathscr{H}$, and its dual $\mathscr{A}$, which can be treated as an algebra of formal distributions on $G$.

We denote by $\hat{G}$ a fixed set of irreducible inequivalent unitary finite dimensional representations of $G$, such that $\hat{G}$ contains one and only one element of each equivalence class.

Let $\rho$ be any finite dimensional representation of $G$, acting on $V_{\rho}$.

(2.1) Definition. Given $M \in \mathscr{L}\left(V_{\rho}\right)$, the coefficient of $\rho$ associated with $M$ is the function $C_{M}^{\rho} \in C^{\infty}(G)$ defined by: $x \in G, C_{M}^{\rho}(x)=\operatorname{Tr}(M \rho(x))$. We denote by $\mathscr{C}_{\rho}$ the space of coefficients of $\rho$.

We note that the algebra structure of the bicommutant of $\rho$ induces an associative algebra structure on $\mathscr{C}_{\rho}$ with unit element $\xi^{\rho}=C_{\mathrm{Id}}^{\rho}$, i.e. the character of $\rho$.

From the Burnside-Schur theorem, $\mathscr{C}_{\rho} \simeq \mathscr{L}\left(V_{\rho}\right)$ if and only if $\rho$ is irreducible.

(2.2) Proposition. Let $\rho$ and $\rho^{\prime}$ be finite dimensional representations of $G$, then $\rho \simeq \rho^{\prime}$ if and only if $\mathscr{C}_{\rho}=\mathscr{C}_{\rho^{\prime}}$ (as algebras), which occurs if and only if $\xi^{\rho}=\xi^{\rho^{\prime}}$.

Proof. Assume $\rho \simeq \rho^{\prime}$ by $f: V_{\rho} \rightarrow V_{\rho^{\prime}}$, then $C_{M}^{\rho^{\prime}}=C_{f^{-1} M f}^{\rho}$, so $\mathscr{C}_{\rho}=\mathscr{C}_{\rho^{\prime}}$, and $\xi^{\rho}=\xi^{\rho^{\prime}}$. On the other hand, if $\mathscr{C}_{\rho}=\mathscr{C}_{\rho^{\prime}}$ as algebras, then their units do coincide, so $\xi^{\rho}=\xi^{\rho^{\prime}}$ which is equivalent to $\rho \simeq \rho^{\prime}$ by e.g. the Peter-Weyl theorem. Q.E.D.

By (2.2) and the Peter-Weyl theorem, the subspace of $C^{\infty}(G)$ generated by coefficients functions of finite dimensional representations of $G$ is exactly $\mathscr{H}=\mathscr{H}(G)=\bigoplus_{\pi \in \hat{G}} \mathscr{C}_{\pi} ;$ note that $\mathscr{C}_{\pi} \simeq \mathscr{L}\left(V_{\pi}\right)$ as algebras, when $\pi \in \hat{G}$. Now $\mathscr{H}$ has another algebra structure, coming from the usual (commutative) multiplication “ $\times$ " in $C^{\infty}(G)$ :

(2.3) Proposition. $\mathscr{H}$ is a subalgebra of $C^{\infty}(G)$. One has:

$$
M \in \mathscr{L}\left(V_{\rho}\right), \quad M^{\prime} \in \mathscr{L}\left(V_{\rho^{\prime}}\right), \quad C_{M}^{\rho} \times C_{M^{\prime}}^{\rho^{\prime}}=C_{M \otimes M^{\prime}}^{\rho \otimes \rho^{\prime}}
$$

Proof. We use the usual identification $\mathscr{L}\left(V_{\rho}\right) \otimes \mathscr{L}\left(V_{\rho^{\prime}}\right) \simeq \mathscr{L}\left(V_{\rho} \otimes V_{\rho^{\prime}}\right)$; then the formula in (2.3) is an obvious application of the properties of the trace. Q.E.D.

(2.4) The Algebra $\mathscr{H}$.

(2.4.1) In the foregoing, $\mathscr{H}=\mathscr{H}(G)$ with its commutative algebra structure inherited from $C^{\infty}(G)$ will be called the algebra of coefficients of $G$. Since $G$ is a compact connected Lie group, $\mathscr{H}$ is of countable dimension (actually, it is known that $\mathscr{H}$ is a finitely generated algebra [4]), and has an obvious well-behaved topological Hopf algebra structure: topology is the natural one (see Appendix 2) and:

(1) Let $\delta$ be the coproduct of $C^{\infty}(G)$ (see Sect.1). Set $C_{i j}^{\pi}=C_{E_{i j}}^{\pi}$, where $E_{i j}=e_{i}^{*} \otimes e_{j},\left\{e_{i}\right\}$ basis of $V_{\pi}$. Then $\delta\left(C_{i j}^{\pi}\right)=\sum_{k} C_{i k}^{\pi} \otimes C_{k j}^{\pi} \in \mathscr{H} \otimes \mathscr{H}$, so the restriction of $\delta$ to $\mathscr{H}$ defines a coproduct on $\mathscr{H}$. 
(2) Let $S$ be the antipode of $C^{\infty}(G)$ (see Sect. 1), then $S\left(C_{i j}^{\pi}\right)=C_{j i}^{\check{\pi}} \in \mathscr{H}$.

(3) The counit of $C^{\infty}(G)$ is a counit for $\mathscr{H}$.

From the definition of the topology of $\mathscr{H}$, the results of Appendix 2 do apply: $\mathscr{H}$ is nuclear, and therefore reflexive (which answers a question of [9]), $\mathscr{H}$ is well-behaved, and the dual $\mathscr{H}^{*}$ is also a well-behaved topological Hopf algebra. We shall next give a complete description of $\mathscr{H}^{*}$. For the time being, let us give another realization of $\mathscr{H}$ : consider the (right or left) regular representation of $G$ in $\mathscr{L}^{2}(G)$; then, from the Peter-Weyl theorem, $\mathscr{H}$ is exactly the space of $G$-finite vectors of the regular representation. $\mathscr{C}_{\pi}, \pi \in \hat{G}$, is an isotypical component (of type $\check{\pi}$ for the left regular, of type $\pi$ for the right regular). So $\mathscr{H}$ is closely related to harmonic analysis on $G$, and the construction of $\mathscr{H}^{*}$, that we shall describe in (2.5), is nothing but the Fourier transform on $G$ (up to normalization coefficients).

(2.4.2). We note that any coefficient is an analytic function on $G$, so any element of $\mathscr{H}$ is an analytic function on $G$, and therefore $\mathscr{H}$ is a domain.

(2.4.3). Since $G$ is compact, there exist finite dimensional irreducible representations $\pi_{1}, \ldots, \pi_{k}$, such that any element $\pi$ of $\hat{G}$ can be obtained as a subrepresentation of tensor products of $\pi_{1}, \ldots, \pi_{k}$. From (2.3) and the proof of (2.2), it follows that the coefficients of $\pi$ are polynomials in the coefficients of $\pi_{1}, \ldots, \pi_{k}$. So $\mathscr{H}$ is a finitely generated algebra. We shall give more details about the choice of $\pi_{1}, \ldots, \pi_{k}$ in Sect. 7 .

(2.5) The Algebra $\mathscr{A}$. Given $\pi \in \hat{G}$, we identify $\mathscr{L}\left(V_{\pi}\right)$ and $\mathscr{L}\left(V_{\pi}\right)^{*}$ by the following duality:

$$
M, M^{\prime} \in \mathscr{L}\left(V_{\pi}\right), \quad\left\langle M \mid M^{\prime}\right\rangle=\operatorname{Tr}\left(M M^{\prime}\right) .
$$

We introduce $\mathscr{A}=\mathscr{A}(G)=\prod_{\pi \in \hat{G}} \mathscr{A}_{\pi}$, where $\mathscr{A}_{\pi}=\mathscr{L}\left(V_{\pi}\right)$, with product topology and associative structure of algebra defined by:

$$
a=\left(a_{\pi}\right), \quad b=\left(b_{\pi}\right), \quad c=\left(c_{\pi}\right)=a * b, \quad \text { with } c_{\pi}=a_{\pi} \circ b_{\pi}, \forall \pi \in \hat{G} .
$$

We get a topological algebra. As a vector space, we have $\mathscr{A}(G)=\mathscr{A}=\mathscr{H}^{*}$, if we define the duality by:

$$
a=\left(a_{\pi}\right) \in \mathscr{A}, \quad \pi_{0} \in \hat{G}, \quad M \in \mathscr{L}\left(V_{\pi_{0}}\right), \quad\left\langle a \mid C_{M}^{\pi_{0}}\right\rangle=\left\langle a_{\pi_{0}} \mid M\right\rangle .
$$

We define a map $i: G \rightarrow \mathscr{A}$ by $i(x)=(\pi(x)) \in \mathscr{A}, x \in G$.

(2.5.4) Lemma. The mapping $i$ is one to one, bicontinuous from $G$ onto $i(G)$, and $\overline{\operatorname{Vect}(i(G))}=\mathscr{A}$.

Proof. Obviously $i$ is continuous, and bicontinuous since $G$ is compact. It is one to one because $\hat{G}$ is a complete set of representations of $G$ (Peter-Weyl). Since $i(G)^{\perp}=\{0\}$, one has $\overline{\operatorname{Vect}(i(G))}=\mathscr{A}$.

Q.E.D.

Henceforth, we identify $G$ and $i(G)$, so we consider that $G \subset \mathscr{A}$.

\section{(2.5.5) Lemma.}

$$
\forall x, x^{\prime} \in G, \quad h \in \mathscr{H}, \quad\left\langle x * x^{\prime}, h\right\rangle=\left\langle x \otimes x^{\prime}, \delta(h)\right\rangle .
$$

Proof. We can restrict to $h=C_{M}^{\pi}, \pi \in \hat{G}, M \in \mathscr{L}\left(V_{\pi}\right)$. Then

$$
\left\langle x * x^{\prime} \mid C_{M}^{\pi}\right\rangle=\operatorname{Tr}\left(\pi(x) \pi\left(x^{\prime}\right) M\right)=\operatorname{Tr}\left(M \pi\left(x x^{\prime}\right)\right)=C_{M}^{\pi}\left(x x^{\prime}\right) . \quad \text { Q.E.D. }
$$


Lemmas (2.5.4) and (2.5.5) prove that our product $*$ on $\mathscr{A}$ is exactly the transpose ${ }^{T} \delta$ of $\delta$. So we achieve the definition of a topological Hopf structure on $\mathscr{A}$ using (1.3). Let us check the coproduct, counit and antipode:

(1) Let $\Delta_{0}={ }^{T} \times$, where $\times$ is the product on $\mathscr{H}$. Then:

$x \in G, \quad\left\langle\Delta_{0}(x) \mid h \otimes h^{\prime}\right\rangle=\left\langle x \mid h h^{\prime}\right\rangle=\langle x \mid h\rangle\left\langle x \mid h^{\prime}\right\rangle=\left\langle x \otimes x \mid h \otimes h^{\prime}\right\rangle$,

so $\Delta_{0}(x)=x \otimes x, x \in G$.

(2) $1_{\mathscr{H}}$ is exactly the trivial representation $\varepsilon$ of $G$ (or $\mathscr{A}$ ).

(3) The antipode $S$ of $\mathscr{A}$ is defined as the mapping $S: \mathscr{A}_{\pi} \rightarrow \mathscr{A}_{\tilde{\pi}}, \pi \in \hat{G}$, given by:

$$
a \in \mathscr{A}_{\pi}=\mathscr{L}\left(V_{\pi}\right), \quad S(a)={ }^{T} a \in \mathscr{L}\left(V_{\pi}^{*}\right)=\mathscr{A}_{\check{\pi}} .
$$

So one has: $a=\left(a_{\pi}\right) \in \mathscr{A}, S(a)=\left((S(a))_{\pi}\right)$ and $S(a)_{\pi}={ }^{T} a_{\check{\pi}}$. When $x \in G$, one finds $S(x)=x^{-1}$.

The topological Hopf structure of $\mathscr{A}=\mathscr{H}^{*}$ is exactly the transpose of the Hopf structure of $\mathscr{H}$, as defined in (1.3). $\mathscr{H}$ and $\mathscr{A}$ being well-behaved, by applying transposition to the Hopf structure of $\mathscr{A}$, one recovers the Hopf structure of $\mathscr{H}($ see $(1.3))$.

(2.6) Further Properties of $\mathscr{A}$. So far we have an explicit form for $\Delta_{0}$ when restricted to $G$. It is of interest to know how $\Delta_{0}(a)$ can be computed for any $a \in \mathscr{A}$. For this purpose, we consider $\mathscr{A} \hat{\otimes} \mathscr{A}=\prod_{\pi, \pi^{\prime} \in \hat{G}} \mathscr{A}_{\pi} \otimes \mathscr{A}_{\pi^{\prime}}$ ((A.1.6)) and identify $\mathscr{A}_{\pi} \otimes \mathscr{A}_{\pi^{\prime}}=\mathscr{L}\left(V_{\pi}\right) \otimes \mathscr{L}\left(V_{\pi^{\prime}}\right)=\mathscr{L}\left(V_{\pi} \otimes V_{\pi^{\prime}}\right)$. Then $\mathscr{A} \hat{\otimes} \mathscr{A}=\prod_{\pi, \pi^{\prime} \in \hat{G}} \mathscr{A}_{\pi, \pi^{\prime}}$, with $\mathscr{A}_{\pi, \pi^{\prime}}=\mathscr{L}\left(V_{\pi} \otimes V_{\pi^{\prime}}\right)$, and product: $a=\left(a_{\pi, \pi^{\prime}}\right), b=\left(b_{\pi, \pi^{\prime}}\right) \in \mathscr{A} \otimes \mathscr{A}$, then $a * b=\left(c_{\pi, \pi^{\prime}}\right)$, with $c_{\pi, \pi^{\prime}}=a_{\pi, \pi^{\prime}} \circ b_{\pi, \pi^{\prime}}$ in $\mathscr{L}\left(V_{\pi} \otimes V_{\pi^{\prime}}\right)$.

As usual, given $\pi, \pi^{\prime} \in \hat{G}$, the tensor product representation $\rho_{\pi, \pi^{\prime}}=\pi \otimes \pi^{\prime}$ is the representation of $G$ in $V_{\pi} \otimes V_{\pi^{\prime}}$ defined by: $\rho_{\pi, \pi^{\prime}}(x)=\pi \otimes \pi^{\prime}\left(\Delta_{0}(x)\right)$, $x \in G$, where $\pi \otimes \pi^{\prime}: \mathscr{A} \otimes \mathscr{A} \rightarrow \mathscr{A}_{\pi, \pi^{\prime}}=\mathscr{L}\left(V_{\pi} \otimes V_{\pi^{\prime}}\right)$ is the canonical projection: $\pi \otimes \pi^{\prime}(a \otimes b)=\pi(a) \otimes \pi^{\prime}(b), a, b \in \mathscr{A}$.

Now $\rho_{\pi, \pi^{\prime}}$ extends to a representation of $\mathscr{A}$ on $V_{\pi} \otimes V_{\pi^{\prime}}$, say $\rho_{\pi, \pi^{\prime}}$, defined exactly by the same formula. So we have:

$$
a \in \mathscr{A}, \quad \Delta_{0}(a)_{\pi, \pi^{\prime}}=\left(\pi \otimes \pi^{\prime}\right)\left(\Delta_{0}(a)\right)=\rho_{\pi, \pi^{\prime}}(a) .
$$

This gives the component of $\Delta_{0}(a)$ on $\mathscr{A}_{\pi, \pi^{\prime}}$. Precisely, since $G$ is compact, we have $V_{\pi} \otimes V_{\pi^{\prime}}=\sum_{\rho \in t\left(\pi, \pi^{\prime}\right)} W_{\rho}$, with $t\left(\pi, \pi^{\prime}\right)$ a finite subset of $\hat{G}$, and $\left.\rho_{\pi \pi^{\prime}}\right|_{W_{\rho}} \simeq n_{\rho} \cdot \rho$, $n_{\rho} \in \mathbb{N}$. Therefore there exists $\alpha_{\rho}: W_{\rho} \rightarrow V_{\rho} \underbrace{\oplus \ldots \oplus}_{n_{\rho}} V_{\rho}=n_{\rho} \cdot V_{\rho}$ such that $\left.\rho_{\pi \pi^{\prime}}\right|_{W_{\rho}}=\alpha_{\rho}^{-1} \circ n_{\rho} \rho \circ \alpha_{\rho} ;$ defining $C_{\pi, \pi^{\prime}}=\sum_{\rho \in t\left(\pi, \pi^{\prime}\right)} \alpha_{\rho}$, and the representation $r_{\pi, \pi^{\prime}}=\sum_{\rho \in t\left(\pi, \pi^{\prime}\right)} n_{\rho} \cdot \rho$, on the space $\bigoplus_{\rho \in t\left(\pi, \pi^{\prime}\right)} n_{\rho} \cdot V_{\rho}$, we find that $\rho_{\pi, \pi^{\prime}}=C_{\pi, \pi^{\prime} \circ}^{-1} r_{\pi, \pi^{\prime}}$ - $C_{\pi, \pi^{\prime}}$, and $\Delta_{0}(a)_{\pi, \pi^{\prime}}$ is explicitly computed.

(2.6.1) Lemma. Let $i: \mathscr{U}(g) \rightarrow \mathscr{A}$ be the linear map defined by $i(u)=(\pi(u)), u \in \mathscr{U}(g)$. Then $i$ is one to one, and $\overline{i(\mathscr{U}(g)}=\mathscr{A}$.

As a consequence, we can consider that $\mathscr{U}(g) \subset \mathscr{A}$ as Hopf algebras (by (2.6)). Another expression of (2.6.1) is the following: $\hat{G}$ is a complete set of (irreducible) finite dimensional representations of $\mathscr{U}(g)$ (this is not completely obvious a priori, 
since $\hat{G}$ is generally not identical with the set of all finite dimensional irreducible representations of $\mathscr{U}(g)$ ).

Proof. Assume that $u \in \mathscr{U}(g)$, and $\pi(u)=0, \forall \pi \in \hat{G}$. Let $\rho$ be the (left) regular representation space of $G$ on $C^{\infty}(G)$, which is a $C^{\infty}$ representation of $G$; then $\rho(u)$ is a differential operator on $G$, and it is well-known that $\rho: \mathscr{U}(g) \rightarrow \operatorname{Diff}(G)$ is one to one ([15]). The space of $G$-finite vectors of $\rho$ is $\mathscr{H}$, and the restriction of $\rho$ to $\mathscr{H}$ reduces as $\left.\rho\right|_{\mathscr{H}}=\sum_{\pi \in \hat{G}}(\operatorname{dim} \pi) \cdot \pi$ (Peter-Weyl); so $\left.\rho(u)\right|_{\mathscr{H}}=0$, and since $\overline{\mathscr{H}}=C^{\infty}(G)$, we deduce that $\rho(u)=0$, and then that $u=0$. For the density, we consider $V=\sum_{\pi \in \hat{G}} V_{\pi}$, endowed with the representation $\bigoplus_{\pi \in \hat{G}} \pi$; this is a semisimple $\mathscr{U}(g)$-module, with irreducible isotypical components $V_{\pi}$. Its bicommutant is $\mathscr{A}$, so, by Jacobson's density theorem, given $v_{1}, \ldots, v_{n} \in V, a \in \mathscr{A}$, we can find $u \in \mathscr{U}$ such that $\bigoplus_{\pi \in \hat{G}} \pi(a)\left(v_{i}\right)=\bigoplus_{\pi \in \hat{G}} \pi(u)\left(v_{i}\right)$, which proves the result. Q.E.D.

(2.6.2) Remark.

(1) One has $\mathscr{H} \subset C^{\infty}(G)$, this injection is continuous and $\overline{\mathscr{H}}=C^{\infty}(G)$. By transposition, we get that $A \subset \mathscr{A}$, and $\bar{A}=\mathscr{A}$ (another proof of (2.5.4)). All these inclusions are compatible with the Hopf structures. So $\mathscr{A}$ is a completion of the Hopf algebra $A$ of distributions on $G$; this is the reason why we call $\mathscr{A}$ the algebra of formal distributions on $G$.

(2) The map $i$ of (2.6.1) is valued in $A$, so $\mathscr{U}(g) \subset A$, as Hopf algebras. However, $\overline{\mathscr{U}(g)} \neq A$.

\section{(2.7) Ideals and Representations of $\mathscr{A}$.}

\section{(2.7.1) Proposition.}

(1) Let I be a left (resp: right) closed ideal of $\mathscr{A}$, then there exists a left (resp: right) closed ideal $J$ such that $\mathscr{A}=I \oplus J$ (topological direct sum).

(2) Let I be a left (resp: right) closed ideal of $\mathscr{A}$, then $I=\prod_{\pi \in \hat{G}}\left(I \cap \mathscr{A}_{\pi}\right)$.

(3) Let I be a two sided closed ideal of $\mathscr{A}$, then there exists a subset $\hat{G}_{I} \subset \hat{G}$ such that:

$$
I=\prod_{\pi \in \hat{G}_{I}} \mathscr{A}_{\pi}
$$

(4) Let $\rho$ be a finite dimensional $\mathscr{A}$-module, then there exists $G_{\rho} \subset \hat{G}$ such that $\rho \simeq \sum_{\pi \in G_{\rho}} n_{\pi} \cdot \pi$, with $n_{\pi} \in \mathbb{N}$; as a consequence $\rho$ is a continuous $\mathscr{A}$-module.

Proof. (1) Using (A.2.9), $I$ has a topological supplement $V$ in $\mathscr{A}$. So, if we set $\rho(x)(a)=x a, x \in G, a \in \mathscr{A}, \bar{\rho}(x)(\bar{a})=\overline{x a}, \bar{a} \in \mathscr{A} / I$, the obtained representation $\rho$ of $G$ is an extension of $\bar{\rho}$ in the sense of [18]. Since the canonical map $\mathscr{A} \rightarrow \mathscr{A} / I$ has a continuous section, such an extension is related to a cohomology, namely to $H^{1}\left(G, \mathscr{L}_{c}(A / I, I)\right)$ (see [18]). But $G$ being compact, $H^{1}$ vanishes ([14]), so the extension splits, i.e., $I$ has a $\rho$-stable topological supplementary, which is the wanted ideal $J$.

(2) First, since $I$ is closed, if $i_{\pi} \in I, \forall \pi \in \hat{G}$, then $\left(i_{\pi}\right) \in I$, so $\prod_{\pi \in \hat{G}}\left(I \cap \mathscr{A}_{\pi}\right) \subset I$. Then, using (1), we write $\mathscr{A}=I \oplus J$, where $J$ is a closed left ideal, and deduce that $1=1_{I}+1_{J}$; if $i \in I$, then $i=i \cdot 1_{I}$.

Let $i=\left(i_{\pi}\right), 1_{I}=\left(1_{I \pi}\right)$, one has $i=\left(i_{\pi} \cdot 1_{I \pi}\right)$, but $i_{\pi} \cdot 1_{I \pi}=1_{\pi} \cdot i \in I$, so the result is proved.

(3) Using (2), $I=\prod_{\pi \in \hat{G}}\left(I \cap \mathscr{A}_{\pi}\right)$, and since $\mathscr{A}_{\pi}=\mathscr{L}\left(V_{\pi}\right)$ is a simple algebra, one has $I \cap \mathscr{A}_{\pi}=\{0\}$ or $\mathscr{A}_{\pi}$. 
(4) Using (2.5.4), the result is trivial if $\rho$ is assumed continuous. To carry out a proof without this assumption, we have to introduce the center $Z(\mathscr{A})$. It is clear that $Z(\mathscr{A})=\prod_{\pi \in \hat{G}} \mathbb{C} 1_{\pi}$. Now $\hat{G}$ is countable, so we can choose a bijection between $\hat{G}$ and $Z$; let us denote by $n_{\pi}$ the integer which corresponds to $\pi \in \hat{G}$, and introduce $Q=\left(n_{\pi} 1_{\pi}\right) \in Z(\mathscr{A})$.

Now, let $\lambda_{1}, \ldots, \lambda_{k}$ be the eigenvalues of $\rho(Q)$, and $V\left(\lambda_{1}\right), \ldots, V\left(\lambda_{k}\right)$ the corresponding generalized eigenspaces; then $V_{\rho}=\bigoplus_{i=1}^{k} V\left(\lambda_{i}\right)$, and each $V\left(\lambda_{i}\right)$ is a sub- $\mathscr{A}$-module, so we can restrict to the case $V_{\rho}=V(\lambda), \lambda \in \mathbb{C}$. Setting $\alpha=\operatorname{dim} V_{\rho}$, one has $(Q-\lambda)^{\alpha} \in \operatorname{Ker} \rho$; if $\lambda \neq n_{\pi}, \forall \pi \in \hat{G}$, then $(Q-\lambda)^{\alpha}$ has an inverse in $\mathscr{A}$, so $\mathscr{A}=\operatorname{ker} \rho$; if $\lambda=n_{\pi_{0}}, \pi_{0} \in \hat{G}$, then $\left(Q-n_{\pi_{0}}\right)^{\alpha} \mathscr{A}=\prod_{\pi \neq \pi_{0}} \mathscr{A}_{\pi} \subset \operatorname{ker} \rho$, so $\rho$ is actually a representation of the simple algebra $\mathscr{A}_{\pi_{0}}=\mathscr{A} / \prod_{\pi \neq \pi_{0}} \mathscr{A}_{\pi}$, and this completes the proof of (4).

Q.E.D.

(2.7.2) Remarks. Let us come back to the center $Z(\mathscr{A})$, and explain some properties of the very special element $Q$ introduced in the proof of (2.7.1) (4). (Actually, $Q$ can be seen as some kind of generalized Casimir, with very nice properties):

(1) First, from the definition of $Q$, a finite dimensional irreducible representation $\rho$ is characterized, up to equivalence, by the number $\rho(Q)$.

(2) $Q$ generates $Z(\mathscr{A})$ in the following sense: given $C=\left(\lambda_{\pi} 1_{\pi}\right) \in \mathscr{A}, \lambda_{\pi} \in \mathbb{C}$, there exists an entire function $f(x)=\sum_{\rho} f_{\rho} z^{\rho}, z \in \mathbb{C}$, such that $f\left(n_{\pi}\right)=\lambda_{\pi}$. Now, the series $f(Q)=\sum_{\rho} f_{\rho} Q^{\rho}$ converges in $\mathscr{A}$, and $C=f(Q)$. So, if we denote by $\mathscr{E}$ the algebra of complex entire functions, by $\mathscr{I}$ the closed ideal generated by $\sin J \pi z$, one has $Z(\mathscr{A}) \simeq \mathscr{E} / I$.

\section{Deformation Theory of Topological Algebras}

Let us first extend the notion of topological algebras, bialgebras, etc. to the $\mathbb{C}[[t]]$ case.

(3.1) Definition. A topological $\mathbb{C}[[t]]$-algebra $\tilde{A}$ is a topologically free $\mathbb{C}[[t]]$ module $\tilde{A} \simeq A[[t]]$, where $A$ is a c.t.v.s., with a $\mathbb{C}[[t]]$-bilinear continuous product $\tilde{\mu}$.

Obviously, $\tilde{\mu}$ induces a product $\mu$ on $A$, endowing $A$ with a topological algebra structure, as defined in (A.4.1). We call $A$ the classical algebra associated to $\widetilde{A}$.

(3.2) Definition. Given a topological algebra $A$, a deformation of $A$ is a topologically free $\mathbb{C}[[t]]$-algebra $\tilde{A}$ (see $(A .3 .2)$ ), such that $\tilde{A} / t \tilde{A} \simeq A$.

The simplest example is the trivial deformation: let $\mu$ be the product of $A$, then $\mu \in \mathscr{L}(A \hat{\otimes} A, A) \subset \mathscr{L}(A \hat{\otimes} A, A)[[t]] \simeq \mathscr{L}_{t}\left(A[[t]] \hat{\otimes}_{t} A[[t]], A[[t]]\right)(\mathrm{A} .3 .1)$ and (A.3.2)), so $\mu$ extends to a continuous $\mathbb{C}[[t]]$-bilinear product on $A[[t]]$.

(3.3) Equivalence. As usual, deformations are equivalent if they are isomorphic as topological $\mathbb{C}[[t]]$-algebras, the isomorphism reducing to the identity modulo $t$, and a deformation is trivial if it is equivalent to the trivial deformation. A deformation $\tilde{A}$ with product $\tilde{\mu}$ of an algebra $A$ with product $\mu$ is completely specified when one knows:

$$
\begin{aligned}
& a, b \in A, \quad \tilde{\mu}(a, b) \stackrel{\text { def }}{=} a \times{ }_{t} b=a \times b+t C_{1}(a, b)+t^{2} C_{2}(a, b)+\ldots, \\
& \text { with } \quad C_{i} \in \mathscr{L}(A \hat{\otimes} A, A) .
\end{aligned}
$$


This looks exactly like the usual algebraic theory [10], except that the cochains $C_{i}$ involved have to be continuous. Note that if $A$ is an algebra of countable dimension, then by (A.2.9), the topological deformation theory of the topological algebra $A$ (see (1.5.1)), and the usual algebraic theory are the same.

(3.4) Topological Deformations and Cohomology. If $A$ is a (general) associative topological algebra, one can restrict to associative deformations, and the usual cohomological machinery can be used, noticing that cohomology in that case is continuous cohomology. So, from (3.2.1), the leading term $C_{1} \in Z_{c}^{2}(A, A)$, obstructions are in $Z_{c}^{3}(A, A)$, etc.. Then, by standard arguments [10] one has:

\section{(3.4.1) Lemma.}

(1) If $H_{c}^{2}(A, A)=\{0\}$, any deformation of $A$ is trivial ( $A$ is rigid).

(2) If $H_{c}^{3}(A, A)=\{0\}$, any cocycle in $Z_{c}^{2}(A, A)$ is the leading term of at least one deformation.

(3.5) Unit. Let us assume that $\tilde{A}$ is a deformation with associative product of a topological associative algebra $A$. We recall that in our terminology, associative means: associative product and existence of unit. Exactly as in the algebraic case (see [10]), it can be shown that $\tilde{A}$ has a unit, so $\tilde{A}$ is an associative deformation, and passing, if necessary, to an equivalent deformation, one may even assume that $1_{\tilde{A}}=1_{A}$.

(3.6) Formal Series. We need to extend our topological notions of bialgebras, Hopf algebras, etc. to $\mathbb{C}[[t]]$-algebras (3.1). This is straightforward, so we give less details: actually in the axioms of (A.4.1), in order to define topological $\mathbb{C}[[t]]$ bialgebras, Hopf algebras, etc., one has to assume:

(1) that $\tilde{A}$ is a topologically free $\mathbb{C}[[t]]$-module, $\tilde{A} \simeq A[[t]]$, where $A$ is a c.t.v.s.

(2) that the mappings involved such as product, coproduct, counit, antipode, are $\mathbb{C}[[t]]$-linear and continuous.

(3) and to replace $\hat{\otimes}$ by $\hat{\otimes}_{t}$ (see (A.3.5.1)).

Given a topologically free $\mathbb{C}[[t]]$-bialgebra $\tilde{A}$, then $A=\tilde{A} / t \tilde{A}$ is a topological bialgebra: actually we can assume that $\tilde{A}=A[[t]]$, as $\mathbb{C}[[t]]$-module, so $\tilde{A} \hat{\otimes}_{t} \tilde{A}=(A \hat{\otimes} A)[[t]]$ then $\mathscr{L}_{t}\left(\tilde{A}, \tilde{A} \hat{\otimes}_{t} \tilde{A}\right) \simeq \mathscr{L}(A, A \hat{\otimes} A)[[t]]$, therefore the coproduct $\tilde{\Delta}$ is completely specified by:

$a \in A, \tilde{\Delta}(a)=\Delta(a)+t D_{1}(a)+t^{2} D_{2}(a)+\ldots$, where $D_{i} \in \mathscr{L}(A, A \hat{\otimes} A)$ and $\Delta$ is the coproduct for $A$.

Similar arguments show that when $\tilde{A}$ is a topological $\mathbb{C}[[t]]$-Hopf algebra, then $A=\tilde{A} / t \tilde{A}$ is a Hopf algebra. Now the notion of deformation is clear:

(3.7) Definition. Given a topological bialgebra (resp: Hopf algebra) A, a deformation of $A$ is a topological $\mathbb{C}[[t]]$-bialgebra (resp: Hopf algebra) $\tilde{A}$, such that $\tilde{A} / t A \simeq A$.

Equivalence is defined as in (3.2), and has to relate respective coproducts, antipode, etc., as usual. The unit and counit of the deformed bialgebra are identical with those of the original. However, while any deformation of a Hopf algebra continues to be Hopf, i.e., will continue to have an antipode, it will generally not be the same as the original. 
(3.8) Proposition. Let $\tilde{A}$ be a bialgebra (resp: Hopf) deformation of a well-behaved topological bialgebra (resp: Hopf) algebra A. Then the $\mathbb{C}[[t]]-d u a l \tilde{A}_{t}^{*}($ see (A.3.3.2)) is a deformation of the topological Hopf algebra $A^{*}$. Deformations $\tilde{A}$ and $\tilde{A}^{\prime}$ of $A$ are equivalent if and only if $\tilde{A}_{t}^{*}$ and $\tilde{A}_{t}^{\prime *}$ are equivalent deformations of $A^{*}$.

Proof. We prove (3.8) in the Hopf case.

The Hopf structure of $A^{*}$ was defined in (1.3). We can assume that $\tilde{A}=A[[t]]$, and $\tilde{A}_{t}^{*}$ stands for $A[[t]]_{t}^{*} \simeq A^{*}[[t]]$, as $\mathbb{C}[[t]]$-modules (A.3.3.3). Let $\tilde{\mu}, \tilde{\Delta}, \tilde{\varepsilon}$ and $\tilde{S}$ be the respective product, coproduct, counit and antipode on $A[[t]]$, which deform the corresponding objects $\mu, \Delta, \varepsilon$ and $S$; we can assume that $1_{\tilde{A}}=1_{A}$ (see (3.5)). Using $\mathbb{C}[[t]]$-transposition, as defined in (A.3.4), and $\left(\tilde{A} \hat{\otimes}_{t} \tilde{A}\right)_{t}^{*}=$ $((A \hat{\otimes} A)[[t]])_{t}^{*} \simeq(A \hat{\otimes} A)^{*}[[t]]=\left(A^{*} \hat{\otimes} A^{*}\right)[[t]]=\tilde{A}_{t}^{*} \otimes_{t} \tilde{A}_{t}^{*}$ (cf. Appendices A1 and A3), we obtain a $\mathbb{C}[[t]]-H o p f$ structure on $\tilde{A}_{t}^{*}$, with respective product, coproduct, counit and antipode defined by: ${ }^{T} \tilde{\Delta},{ }^{T} \tilde{\mu},{ }^{T} 1_{A}$, and ${ }^{T} \tilde{S}$; the unit of $\tilde{A}_{t}^{*}$ is ${ }^{T} \tilde{\varepsilon}$.

Now, the Hopf structure of $A^{*}$ is defined exactly in the same way, using usual transposition; so $\tilde{A}_{t}^{*}$ deforms $A^{*}$.

The same transposition argument and $A^{* *}=A$ proves the last claim. Q.E.D.

(3.9) Example. Let $A$ be a countable dimensional Hopf algebra. Then, as shown in (1.5.1) $A$ is a well-behaved topological algebra for its natural topology. So (3.8) applies to this case. As noticed in (3.3.1), algebraic deformation theory [10] of $A$, or (topological) deformation theory are the same. Using (3.8), deformation theory of $A$ is the same as (topological) deformation of $A^{*}$; unless $\operatorname{dim} A<\infty$, this is generally not identical to algebraic deformation theory of $A^{*}$. This is a rather striking example of how continuity can be hidden in an, a priori, purely algebraic problem!

(3.10) Remark. Let $A$ be a Hopf algebra; it is proved in [12] that any associative and coassociative bialgebra algebraic deformation of $A$ is actually a Hopf algebra. The same results holds in the topological case, and the proof is essentially the same.

\section{Deformations of the Topological Hopf Algebras of a Compact Lie Group}

In this section, we study the properties of deformations, as defined in Sect. 3, of the Hopf algebras $\mathscr{H}=\mathscr{H}(G)$, or $H=H(G)=C^{\infty}(G)$, or, equivalently, (see (3.8)), of their respective dual algebras $\mathscr{H}^{*}=\mathscr{A}(G)=\mathscr{A}$, or $H^{*}=A(G)=A$. From (3.9), deformation theory of $\mathscr{H}$ is equivalent to algebraic deformation theory. Our notion of (topological) deformations makes it possible to study deformations of $H$ or $A$; we have not heard that any algebraic theory was tried in that case.

(4.1) Deformations of Representations. We shall need some results about deformations of representations of $G$, that we now introduce (see [17] for more details):

Given a t.v.s. $V, \mathscr{L}_{t}(V[[t]])$ is an algebra, so the isomorphism of (A.3.3.1) defines an algebra structure on $\mathscr{L}(V)[[t]]$. More precisely, one has:

$$
\sum_{n} t^{n} T_{n}, \sum_{n} t^{n} U_{n} \in \mathscr{L}(V)[[t]], \quad\left(\sum_{n} t^{n} T_{n}\right) \circ\left(\sum_{n} t^{n} U_{n}\right) \stackrel{\text { def }}{=} \sum_{n} t^{n}\left(\sum_{i=0}^{n} T_{i} \circ U_{n-i}\right) .
$$

Now, it is obvious that $\sum_{n} t^{n} T_{n}$ has an inverse if and only if $T_{0}$ has an inverse in $\mathscr{L}(V)$. 
(4.1.1) Definition [17]. Given a continuous representation $\pi$ of $G$ in $V$, a deformation (or formal representation) $\tilde{\pi}$ of $\pi$ is a morphism $\tilde{\pi}: G \rightarrow \mathscr{L}(V)[[t]]$ such that

(1) $\tilde{\pi}=\pi+\sum_{n \geqq 1} t^{n} \pi_{n}$

(2) $(g, v) \rightarrow \pi_{n}(g)(v)$ is continuous from $G \times V$ into $V, \forall n$.

Deformations $\tilde{\pi}$ and $\tilde{\pi}^{\prime}$ of $\pi$ are equivalent if there exists $\phi=\mathrm{Id}+$ $\sum_{n \geqq 1} t^{n} \phi_{n} \in \mathscr{L}(V)[[t]]$ such that $\tilde{\pi}^{\prime}=\phi \circ \tilde{\pi} \circ \phi^{-1}$. A deformation $\tilde{\pi}$ of $\pi$ is trivial if it is equivalent to $\pi$.

Assume that $\underset{\sim}{\operatorname{dim}} V<\infty$. We extend the trace map $\operatorname{Tr}: \mathscr{L}(V) \rightarrow \mathbb{C}$ to a $\mathbb{C}[[t]]$ linear trace map $\tilde{\mathrm{Tr}} \mathrm{\mathscr {L }}(V)[[t]] \rightarrow \mathbb{C}[[t]]$, defined by $\tilde{\mathrm{Tr}}=\operatorname{Id}_{\mathbb{C}[[t]]} \otimes \operatorname{Tr}$. Formula $\tilde{\operatorname{Tr}}(\tilde{A} \circ \tilde{B})=\tilde{\operatorname{Tr}}(\tilde{B} \circ \tilde{A})$ is still valid for $\tilde{A}, \tilde{B} \in \mathscr{L}(V)[[t]]$.

Given a continuous representation $\pi$ of $G$ in $V$, and a deformation $\tilde{\pi}$ of $\pi$, we can still define coefficients of $\tilde{\pi}$ by:

$$
\tilde{M} \in \mathscr{L}(V)[[t]], C_{\tilde{M}}^{\tilde{\pi}}(x)=\tilde{\operatorname{Tr}}(\tilde{M} \circ \tilde{\pi}(x)) \in \mathbb{C}[[t]] .
$$

The formal character of $\tilde{\pi}$ is:

$$
\xi^{\tilde{\pi}}=C_{\mathbf{I d}}^{\tilde{\pi}} .
$$

Now $G$ is compact, so representations are rigid:

(4.1.4) Lemma. If $\tilde{\pi}$ is a deformation of a continuous representation $\pi$ of $G$ in a t.v.s. $V$, then $\tilde{\pi}$ is a trivial deformation.

Proof (see [17]). We define $\phi \in \mathscr{L}(V)[[t]]$ by $\phi=\int_{G} \tilde{\pi}(x) \pi\left(x^{-1}\right) d x$; one has $\phi_{0}=\mathrm{Id}_{V}$, and due to the invariance of the Haar measure $\tilde{\pi} \circ \phi=\phi \circ \pi$. Q.E.D.

Assuming once more that $\operatorname{dim} V<\infty$, and setting $\tilde{\pi}=\phi \circ \pi \circ \phi^{-1}$, $\phi \in \mathscr{L}(V)[[t]]$, which is, from (4.1.4), the general case of deformations of $\pi$, one has: given $\tilde{M} \in \mathscr{L}(V)[[t]], C_{\tilde{M}}^{\tilde{\pi}}=C_{\phi^{-1} \circ \tilde{M} \circ \phi}^{\pi}$; let $\tilde{N}=\phi^{-1} \circ \tilde{M} \circ \phi=\sum t^{n} N_{n}$, one has $C_{\tilde{M}}^{\tilde{\pi}}=\sum t^{n} C_{N_{n}}^{\pi}$, so (see Sect. 2):

$$
\begin{gathered}
C_{\tilde{M}}^{\tilde{\tilde{M}}} \in \mathscr{H}[[t]] \text {, and } \\
\xi^{\tilde{\pi}}=\xi^{\pi} .
\end{gathered}
$$

(4.2) Deformations of the Hopf Algebras $\mathscr{A}$ and $A$

(4.2.1) Proposition. Any associative deformation of the algebras $\mathscr{A}$ or $A$ is trivial.

Proof. By (3.4.1) the result is contained in the following lemma:

(4.2.2) Lemma. $H_{c}^{n}(\mathscr{A}, \mathscr{A})=H_{c}^{n}(A, A)=\{0\}, \forall n \geqq 1$.

Proof for $\mathscr{A}$. Let $f=\prod_{k} f_{k} \in Z_{c}^{n}(\mathscr{A}, \mathscr{A})$, then each $f_{k} \in Z_{c}^{n}(\mathscr{A}, \mathscr{A})$; for fixed $k$, we set $\mathscr{A}=\mathscr{A}^{\prime} \oplus \mathscr{A}_{k}, 1_{\mathscr{A}}=1^{\prime}+1_{\mathscr{A}_{k}}, a=a^{\prime}+a_{k}$, for $a \in \mathscr{A}$ and we get:

$$
\begin{aligned}
f\left(a_{1}, \ldots, a_{n}\right)= & f\left(a_{1}^{\prime}, a_{2}, \ldots, a_{n}\right)+f\left(a_{1 k}, a_{2}^{\prime}, a_{3}, \ldots, a_{n}\right) \\
& +f\left(a_{1 k}, a_{2 k}, a_{3}^{\prime}, a_{4}, \ldots, a_{n}\right)+\ldots+f\left(a_{1 k}, \ldots, a_{n-1 k}, a_{n}^{\prime}\right) \\
& +f\left(a_{1 k}, \ldots, a_{n k}\right) .
\end{aligned}
$$

First $f_{k} \mid \otimes_{n} \mathscr{A}_{k}=d\left(\varphi_{k}\right)$, with $\varphi_{k} \in \mathscr{L}^{n-1}\left(\mathscr{A}_{k}, \mathscr{A}_{k}\right)$, since $H^{n}\left(\mathscr{A}_{k}, \mathscr{A}_{k}\right)=\{0\}$. Then, on $\mathscr{A}_{k} \otimes \cdots \otimes \mathscr{A}_{k} \otimes \mathscr{A}_{s}^{\prime} \otimes \mathscr{A} \otimes \cdots \otimes \mathscr{A}$, we introduce $\psi_{k}^{s}\left(x_{1 k}, \ldots, x_{s-1 k}, x_{s}^{\prime}\right.$, $\left.x_{s+1}, \ldots, x_{n-1}\right)=(-1)^{s} f_{k}\left(x_{1 k}, \ldots, x_{s-2 k}, 1^{\prime}, x_{s-1 k}, \quad x_{s}^{\prime}, x_{s+1}, \ldots, x_{n-1}\right), \quad \theta_{k}=$ 
$\sum_{s=1}^{n-1} \psi_{k}^{s}+\varphi_{k}$, and using cocycle relation, we see that $f_{k}=d\left(\theta_{k}\right)$; then, if $\xi=\prod_{k} \theta_{k}$, one has $f=d \xi$. Moreover, from the definition of $\xi$, and the continuity of $f, \xi$ is continuous.

Q.E.D.

(4.2.3) Remarks.

(1) By the same proof, $H^{n}(\mathscr{A}, \mathscr{A})=\{0\}, n \geqq 1$, so, as a consequence, $\mathscr{A}$ is also rigid in the algebraic [10] sense.

(2) By arguments similar to the proof of (4.2.2), one has:

$$
H^{1}(\mathscr{A}, \mathscr{A} \hat{\otimes} \mathscr{A})=H_{c}^{1}(\mathscr{A}, A \hat{\otimes} \mathscr{A})=\{0\} .
$$

It results from [2], that for the cohomology of bialgebras $H_{b i}$ defined in [2], one has $H_{b i}^{2}(\mathscr{A}, \mathscr{A})=H_{b i, c}^{2}(\mathscr{A}, \mathscr{A})=\{0\}$, so $\mathscr{A}$ is rigid in the category of bialgebras, in the sense defined in [2].

Proof for $A$. First, we note that $\mathscr{L}\left(\hat{\otimes}_{n} A, A\right) \simeq\left(\hat{\otimes}_{n} A\right)^{*} \hat{\otimes} A=A\left(G^{n}\right)^{*} \hat{\otimes} A \simeq$ $C^{\infty}\left(G^{n}\right) \hat{\otimes} A=C^{\infty}\left(G^{n}, A\right)$, so given $c \in \mathscr{L}\left(\hat{\otimes}_{n} A, A\right)$, the restriction $\left.c\right|_{G^{n}} \in C^{\infty}\left(G^{n}, A\right)$, and conversely, given $c \in C^{\infty}\left(G^{n}, A\right)$, then $c$ is the restriction of an element of $\mathscr{L}\left(\hat{\otimes}_{n} A, A\right)$.

Now, if $\xi \in Z_{c}^{n}(A, A)$, we set $\theta\left(x_{1}, \ldots, x_{n}\right)=\xi\left(x_{1}, \ldots, x_{n}\right) x_{n}^{-1} \ldots x_{1}^{-1}$, and get $\theta \in Z_{\infty}^{n}(G, A)$ (see [14]), where $G$ acts on $A$ by the representation: $T_{x}(a)=x^{-1}, x \in G, a \in A$. Since $G$ is compact, one has $H_{\infty}^{n}(G, A)=\{0\}$ [14], so there exists $L \in C^{\infty}\left(G^{n-1}, A\right)$ such that $\theta=d_{G}(L)$; but $L \in \mathscr{L}\left(\hat{\otimes}_{n-1} A, A\right)$, so $\xi \in B_{c}^{n}(A, A)$.

Q.E.D.

(4.2.4) Proposition. Let $(\mathscr{A}[[t]], \tilde{\Delta})(\mathrm{resp}:(A[[t]], \tilde{\Delta}))$ be an associative deformation of the bialgebra $\mathscr{A}$ (resp: $A$ ); using (4.2.1), we assume that the product is unchanged. Then there exists $\tilde{P} \in \mathscr{A} \hat{\otimes} \mathscr{A}[[t]]$ (resp: $A \hat{\otimes} A[[t]]$ ) such that $\tilde{\Delta}=\tilde{P} \Delta_{0} \tilde{P}^{-1}$.

Proof. $\tilde{\Delta} \in \mathscr{L}_{t}\left(\mathscr{A}[[t]], \mathscr{A}[[t]] \hat{\otimes}_{t} \mathscr{A}[[t]]\right) \simeq \mathscr{L}(\mathscr{A}, \mathscr{A} \hat{\otimes} \mathscr{A})[[t]] \quad$ (see (A.3.3.1) and (A.3.5.1)); write $\tilde{\Delta}=\Delta_{0}+\sum_{n \geqq 1} t^{n} \Delta_{n}$, and introduce $\tilde{\Delta}(x)=\sum_{n} t^{n} \Delta_{n}(x) \epsilon$ $(\mathscr{A} \hat{\otimes} \mathscr{A})[[t]], x \in G$, and $\tilde{P}=\int_{G} \tilde{\Delta}(x) \Delta_{0}(x)^{-1} d x \in(\mathscr{A} \hat{\otimes} \mathscr{A})[[t]] ;$ due to the invariance of the Haar measure, one has: $\tilde{P} \Delta_{0}(y)=\tilde{\Delta}(y) \tilde{P}, \forall y \in G$, and since $\overline{\operatorname{Vect}(G)}=\mathscr{A}$, one gets $\Delta=\tilde{P} \Delta_{0} \tilde{P}^{-1}$ (note that $\tilde{P}^{-1}$ exists since $\tilde{P}_{0}=1 \otimes 1$ ).

The proof is the same in the case of $A$.

Q.E.D.

Remark. The explicit integral formula for the twist $\tilde{P}=\int_{G} \tilde{\Delta}(x) \Delta_{0}(x)^{-1} d x$ is of interest. Note that for any $\tilde{H} \in(\mathscr{A} \hat{\otimes} \mathscr{A})[[t]]$, or $\left(A \hat{\otimes}_{\tilde{Q}} A\right)[[t]]$, with $\tilde{H}_{0}=1 \otimes 1$, $\tilde{Q}=\int_{G} \tilde{\Delta}(x) \tilde{H} \Delta_{0}(x)^{-1} d x$ also defines a twist between $\tilde{\Delta}$ and $\Delta_{0}$.

(4.2.5) Corollary. Any associative deformation of the bialgebra $\mathscr{A}$ (resp: A) is quasi-cocommutative and quasi-coassociative.

Proof. In the terminology of [7], $\tilde{\Delta}$ is obtained from $\Delta_{0}$ from twisting by $\tilde{P}$, so, using [7] and the fact that $\Delta_{0}$ is cocommutative and coassociative, $\tilde{\Delta}$ is quasicocommutative and quasi-coassociative.

Q.E.D.

(4.2.6) Proposition. Let $(\mathscr{A}[[t]], \tilde{\Delta})(\mathrm{resp}:(A[[t]], \tilde{\Delta}))$ be an associative and coassociative deformation of the algebra $\mathscr{A}$ (resp: $A$ ), with unchanged product. Then the 
counit $\varepsilon$ of $\mathscr{A}$ (resp: $A$ ) is still a counit of $\mathscr{A}[[t]]$ (resp: $A[[t]]$ ), and there exists an antipode $\tilde{S}$, so $\mathscr{A}[[t]]$ (resp: $A[[t]]$ ) is a $\mathbb{C}[[t]]-H o p f$ algebra. Let $S_{0}$ be the antipode of $\mathscr{A}$ (resp: $A$ ), then there exists $\tilde{a} \in \mathscr{A}[[t]]$ such that $\tilde{S}=\tilde{a} S_{0} \tilde{a}^{-1}$.

Proof. As noted in (3.5), the corresponding deformation $\mathscr{H}[[t]]$ of $\mathscr{H}$ (see (3.8)) has a unit $\tilde{\varepsilon}$, which satisfies $\delta(\tilde{\varepsilon})=\tilde{\varepsilon} \otimes \tilde{\varepsilon}$; So $\tilde{\varepsilon}(x y)=\tilde{\varepsilon}(x) \tilde{\varepsilon}(y), \forall x, y \in G$, and this proves that $\left.\tilde{\varepsilon}\right|_{G}$ is a deformation of the trivial representation $\left.\varepsilon\right|_{G}$. By (4.1.4), $\left.\tilde{\varepsilon}\right|_{G}=\left.\varepsilon\right|_{G}$, so $\tilde{\varepsilon}=\varepsilon$.

It was noted in (3.10) that $\mathscr{A}[[t]]$ has an antipode $\tilde{S}$, which deforms $S_{0}$. For any representation $\pi \in \hat{G}, \tilde{\tilde{\pi}}={ }^{T} \pi \circ \tilde{S}$ is a deformation of $\check{\pi}$; using (4.1.4), there exists $\tilde{a}_{\pi} \in \mathscr{L}\left(V_{\pi}^{*}\right)[[t]]$ such that $\tilde{\tilde{\pi}}=\tilde{a}_{\pi} \tilde{\pi} \tilde{a}_{\pi}^{-1}$, so ${ }^{T} \tilde{\tilde{\pi}}=\pi \circ \tilde{S}=\left({ }^{T} \tilde{a}_{\pi}\right)^{-1}(\pi \circ S)^{T} \tilde{a}_{\pi}$; let $\tilde{a}=\left({ }^{T} a_{\pi}\right)^{-1} \in \mathscr{A}[[t]]$, then $\tilde{S}=\tilde{a} S_{0} \tilde{a}^{-1}$.

Similarly, by (3.10), $A[[t]]$ has an antipode $\tilde{S}$ which deforms $S_{0}$.

Let $\tilde{a}=\int_{G} \tilde{S}(x) S_{0}\left(x^{-1}\right) d x \in A[[t]]$; using the (right) invariance of the Haar measure, and the antihomomorphism property of antipodes, one deduces that $\tilde{a} S_{0}(y)=\tilde{S}(y) \tilde{a}, \forall y \in G$; moreover $\tilde{a}(0)=1$, so $\tilde{a}$ has an inverse in $A[[t]]$, and using the density of $G$ in $A$, we get:

$$
\tilde{S}(a)=\tilde{a} S_{0}(a) \tilde{a}^{-1}, \quad \forall a \in A .
$$

Q.E.D.

(4.3) Dualization to $\mathscr{H}$. The results of (4.2) translate by $\mathbb{C}[[t]]$-duality (3.8) as follows:

(4.3.1) Proposition. Let $(\mathscr{H}[[t]], \tilde{x}, \tilde{\delta})$ (resp: $(H[[t]], \tilde{x}, \tilde{\delta}))$ be a coassociative deformation of the bialgebra $\mathscr{H}$ (resp: $H$ ); then, up to equivalence, it can be assumed that $\tilde{\delta}=\delta$. The product is quasi-commutative, and quasi-associative, the counit unchanged. If the product is associative, then $\mathscr{H}[[t]]$ (resp: $H[[t]]$ ) is a $\mathbb{C}[[t]]$ Hopf algebra, with the same unit and counit than $\mathscr{H}$ (resp: $H$ ).

(4.3.1.1) Definition. $A$ deformation of the bialgebra $\mathscr{H}$ (resp: $H$ ) with unchanged coproduct will be called a preferred deformation of $\mathscr{H}$ (resp. $H$ ).

Let us now develop consequences of (4.2.4). We need some notations. Given $\pi$ and $\pi^{\prime} \in \hat{G}$, we denote by $\rho_{\pi, \pi^{\prime}}$ their tensor product $\rho_{\pi, \pi^{\prime}}(x)=\pi \otimes \pi^{\prime}\left(\Delta_{0}(x)\right), x \in G$, and by $\tilde{\rho}_{\pi \pi^{\prime}}$ their new tensor product given by $\tilde{\rho}_{\pi \pi^{\prime}}(x)=\pi \otimes \pi^{\prime}(\tilde{\Delta}(x))$. Using (4.2.4), one has:

$$
\tilde{\rho}_{\pi \pi^{\prime}}=\pi \otimes \pi^{\prime}(\tilde{P}) \rho_{\pi \pi^{\prime}} \pi \otimes \pi^{\prime}(\tilde{P})^{-1}
$$

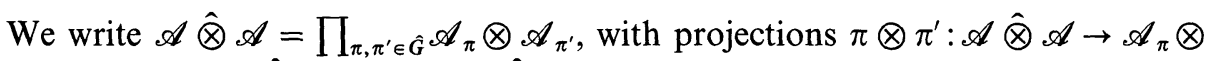
$\mathscr{A}_{\pi^{\prime}}$, and $\mathscr{A}[[t]] \hat{\otimes}_{t} \mathscr{A}[[t]]=(\mathscr{A} \otimes \mathscr{A})[[t]]=\prod_{\pi, \pi^{\prime} \in \hat{G}}\left(\mathscr{A}_{\pi} \otimes \mathscr{A}_{\pi^{\prime}}\right)[[t]]$, with the same projections.

Likewise, for elements $P \in \mathscr{A} \hat{\otimes} \mathscr{A}$ (resp: $\left.\tilde{P} \in \mathscr{A}[[t]] \hat{\otimes}_{t} \mathscr{A}[[t]]\right)$ we write $P=\left(P_{\pi \pi^{\prime}}\right)$, with $P_{\pi \pi^{\prime}}=\pi \otimes \pi^{\prime}(P) \in \mathscr{A}_{\pi} \otimes \mathscr{A}_{\pi^{\prime}} \quad\left(\right.$ resp. $\quad\left(\tilde{P}=\tilde{P}_{\pi \pi^{\prime}}\right)$, with $\tilde{P}_{\pi \pi^{\prime}}=$ $\left.\pi \otimes \pi^{\prime}(P) \in\left(\mathscr{A}_{\pi} \otimes \mathscr{A}_{\pi^{\prime}}\right)[[t]]\right)$.

With these notations, we have:

$$
\rho_{\pi \pi^{\prime}}(x)=\Delta_{0}(x)_{\pi \pi^{\prime}}, \quad \tilde{\rho}_{\pi \pi^{\prime}}(x)=\tilde{\Delta}(x)_{\pi \pi^{\prime}}=\tilde{P}_{\pi \pi^{\prime}} \Delta_{0}(x)_{\pi \pi^{\prime}} \tilde{P}_{\pi \pi^{\prime}}^{-1}
$$


Then, given $M \in \mathscr{L}\left(V_{\pi}\right)$, and $M^{\prime} \in \mathscr{L}\left(V_{\pi^{\prime}}\right)$, we compute, using the trace (as defined in (4.1.1)):

$$
\begin{aligned}
x \in G, C_{M}^{\pi} \tilde{x} C_{M^{\prime}}^{\pi^{\prime}}(x) & =\left\langle C_{M}^{\pi} \otimes C_{M^{\prime}}^{\pi^{\prime}} \mid \tilde{\Delta}(x)\right\rangle \in \mathbb{C}[[t]] \\
& =\left\langle C_{M}^{\pi} \otimes C_{M^{\prime}}^{\pi^{\prime}} \mid \tilde{\Delta}^{\prime}(x)_{\pi \pi^{\prime}}\right\rangle \\
& =\left\langle C_{M}^{\pi} \otimes C_{M^{\prime}}^{\pi^{\prime}} \mid \tilde{\rho}_{\pi, \pi^{\prime}}(x)\right\rangle \\
& =\tilde{T} r\left(M \otimes M^{\prime} \circ \tilde{\rho}_{\pi \pi^{\prime}}(x)\right\rangle=C_{M \otimes M^{\prime}}^{\tilde{\rho}_{\pi \pi^{\prime}}}(x) .
\end{aligned}
$$

So we have proved that $(2.3)$ is valid for preferred deformations, namely:

(4.3.2) Proposition. Let $(\mathscr{H}[[t]], \tilde{x})$ be a preferred deformation of $\mathscr{H}$, then if $\pi, \pi^{\prime} \in \hat{G}, M \in \mathscr{L}\left(V_{\pi}\right), M^{\prime} \in \mathscr{L}\left(V_{\pi^{\prime}}\right), C_{M}^{\pi} \tilde{\times} C_{M^{\prime}}^{\pi^{\prime}}=C_{M \otimes M^{\prime}}^{\pi \tilde{\otimes} \pi^{\prime}}$, where $\pi \tilde{\otimes} \pi^{\prime}=\tilde{\rho}_{\pi \pi^{\prime}}$ stands for the new tensor product of the representations $\pi$ and $\pi^{\prime}$ of $G$.

(4.3.3) Corollary. Under the same assumptions, let $\mathscr{H}_{c}$ be the subalgebra of central functions; then the product on $\mathscr{H}_{c}$ is unchanged.

Proof. $\mathscr{H}_{c}$ is linearly generated by the characters $\xi^{\pi}$ of the elements of $\hat{G}$, so we compute:

$$
\begin{gathered}
\xi^{\pi} \tilde{\times} \xi^{\pi^{\prime}}=C_{\mathrm{Id} V \pi^{\prime}}^{\pi} \tilde{\times} C_{\mathrm{Id} V \pi^{\prime}}^{\pi^{\prime}}=C_{\mathrm{Id} V \pi \otimes \operatorname{ld} V \pi^{\prime}}^{\pi \tilde{\otimes} \pi^{\prime}}=C_{\mathrm{Id}\left(V \pi \pi^{\prime} \otimes V \pi^{\prime}\right)}^{\pi \tilde{\otimes} \pi^{\prime}}=\xi^{\pi \tilde{\otimes} \pi^{\prime}}=\xi^{\pi \otimes \pi^{\prime}} \\
=\xi^{\pi} \times \xi^{\pi^{\prime}} .
\end{gathered}
$$

This raises a natural question: do (4.3.2) and (4.3.3) hold if $\mathscr{H}$ is replaced by $H$ ? Actually, the answer is yes, and this will be a consequence of the following proposition:

(4.3.4) Proposition. If $(H, \tilde{x})$ is a preferred deformation of $H$, then it defines, by restriction, a preferred deformation of $\mathscr{H}$, i.e.:

$$
\forall h, \quad h^{\prime} \in \mathscr{H}, \quad h \tilde{\times} h^{\prime} \in \mathscr{H}[[t]] .
$$

Proof. The inclusion $\mathscr{H} \subset H$ is a (continuous) injective morphism, with dense range; moreover, the coproduct of $\mathscr{H}$ is the restriction of the coproduct of $H$. Therefore, using transposition we obtain a continuous injective morphism $A=H^{*} \mapsto \mathscr{A}=\mathscr{H}^{*}$, with dense range. So we can consider that $A$ is a subalgebra of $\mathscr{A}$; moreover, since the product of $\mathscr{H}$ is the restriction of the product of $H$, the coproduct of $A$ is the restriction of the coproduct $\Delta_{0}$ of $\mathscr{A}$. Using (3.8), the given preferred deformation of $H$ induces a corresponding deformation of $A$, with unchanged product, and coproduct $\tilde{\Delta}=\tilde{P} \Delta_{0} \tilde{P}^{-1}, \tilde{P} \in(A \otimes \hat{\otimes} A)[[t]]$, by (4.2.4). Since $\widetilde{P} \in(\mathscr{A} \hat{\otimes} \mathscr{A})[[t]], \tilde{\Delta}$ is actually a coproduct on $\mathscr{A}$.

Now, given $h, h^{\prime} \in \mathscr{H}$, we write $\tilde{\Delta}=\sum t^{n} \Delta_{n}$, and:

$$
d \in A, \quad\left\langle h \tilde{\times} h^{\prime} \mid d\right\rangle=\left\langle h \otimes h^{\prime} \mid \tilde{\Delta}(d)\right\rangle=\sum t^{n}\left\langle h \otimes h^{\prime} \mid \Delta_{n}(d)\right\rangle=\sum t^{n} C_{n}\left(h, h^{\prime}\right)(d) .
$$

Actually, $C_{n}\left(h, h^{\prime}\right)$ is an element of $H$, completely determined by its restriction to $G \subset A$. On the other hand, the formula $D_{n}\left(h, h^{\prime}\right)(a)=\left\langle h \otimes h^{\prime} \mid \Delta_{n}(a)\right\rangle$ defines an element of $\mathscr{H}$, also completely determined by its restriction to $G$. Since $C_{n}\left(h, h^{\prime}\right)$ and $D_{n}\left(h, h^{\prime}\right)$ coincide on $G$, one has $C_{n}\left(h, h^{\prime}\right)=D_{n}\left(h, h^{\prime}\right) \in \mathscr{H}$, so:

$$
h \tilde{\times} h^{\prime}=\sum_{n} t^{n} C_{n}\left(h, h^{\prime}\right) \in \mathscr{H}[[t]] .
$$

(4.3.5) Corollary. (4.3.2) and (4.3.3) hold with $H$ replacing $\mathscr{H}$. 
Proof. Let $H_{c}$ be the subalgebra (in $H$ ) of central functions, then $\overline{\mathscr{H}}_{c}=H_{c}$, so using (4.3.4), (4.3.3) and the continuity of $C_{n}$ in the development of the product

$$
h \tilde{\times} h^{\prime}=\sum t^{n} C_{n}\left(h, h^{\prime}\right), \quad h, h^{\prime} \in H,
$$

we get (4.3.3) for $H$.

Q.E.D.

(4.3.6) Proposition. Let $\tilde{x}$ be a preferred associative deformation of $H$. By (4.3.1), it is a Hopf deformation, with antipode $\tilde{S}$. Then $\tilde{S}$ restricts to $\mathscr{H}$, i.e.:

$$
\forall h \in \mathscr{H}, \quad \tilde{S}(h) \in \mathscr{H}[[t]],
$$

so the restriction of a Hopf deformation of $H$ defines a Hopf deformation of $\mathscr{H}$.

Proof. Using (4.3.4), the restriction defines a preferred associative deformation of the bialgebra $\mathscr{H}$. From (4.3.1), the unit and counit of $H$ are unchanged, so do restrict to $\mathscr{H}$. Again by (4.3.1), the obtained deformation of $\mathscr{H}$, being associative, is a Hopf deformation, so it has an antipode. The problem is to show that this antipode is the restriction of the antipode of $H$, and this will be done (using unicity of antipode) by showing that $\tilde{S}$ restricts to $\mathscr{H}$. We use $\mathbb{C}[[t]]$-duality (3.8): we have $A=H^{*} \subset \mathscr{A}=\mathscr{A}^{*}$, and our preferred deformation leads to deformations of $A$ and $\mathscr{A}$, with unchanged product, counit and same coproduct (see (4.3.4)). Both have antipode, say $\tilde{s}$ and $\tilde{\mathscr{S}}$, and we want to show that $\left.\tilde{\mathscr{S}}\right|_{A}=\tilde{s}$. By (4.2.6), $\tilde{s}=\tilde{a} S_{0} \tilde{a}^{-1}$, with $\tilde{a} \in A[[t]]$, so $\tilde{s}$ extends to an antipode on $\mathscr{A}[[t]]$, defined by the same formula, and the unicity of the antipode proves that this extension is exactly $\tilde{\mathscr{S}}$.

Q.E.D.

\section{Quotient Deformations}

Given a compact connected Lie group $G$, we continue to denote by $\mathscr{H}(G)$ the algebra of coefficients of $G$, by $H(G)=C^{\infty}(G)$ the algebra of $C^{\infty}$ functions on $G$, and by $\mathscr{A}(G)$ and $A(G)$ their respective duals. We recall that $\mathscr{H}(G \times G) \simeq$ $\mathscr{H}(G) \otimes \mathscr{H}(G)$, and $H(G \times G) \simeq H(G) \hat{\otimes} H(G) ;$ by $(\mathrm{A} .1 .5), \mathscr{A}(G \times G) \simeq \mathscr{A}(G) \hat{\otimes}$ $\mathscr{A}(G)$, and $A(G \times G) \simeq A(G) \hat{\otimes} A(G)$.

Let $\Gamma$ be a normal subgroup of $G$; we introduce $H(G)^{\Gamma}=\{f \in H(G) / f(x \gamma)=$ $f(x), \forall x \in G, \gamma \in \Gamma\}$, and $\mathscr{H}(G)^{\Gamma}=\mathscr{H}(G) \cap H(G)^{\Gamma}$, which are subalgebras respectively of $H(G)$ and $\mathscr{H}(G)$, stable by the antipode since $\Gamma$ is normal.

Now, there is an obvious isomorphism $\phi: H(G)^{\Gamma} \simeq H(G / \Gamma)$, which induces on $H(G)^{\Gamma}$ the coproduct of $H(G / \Gamma)$; one has $H(G)^{\Gamma} \hat{\otimes} H(G)^{\Gamma} \simeq H(G / \Gamma \times G / \Gamma) \simeq$ $H(G \times G / \Gamma \times \Gamma)$, and since $\Gamma$ is normal, this shows that the restriction of the coproduct of $H(G)$ to $H(G)^{\Gamma}$ is exactly the coproduct of $H(G)^{\Gamma}$, so that $H(G / \Gamma) \simeq H(G)^{\Gamma}$ is a Hopf subalgebra of $H(G)$.

Let us show that the same result holds when $H$ is replaced by $\mathscr{H}$. First we note that any element $\pi$ of $\hat{G / \Gamma}$ is actually an element of $\hat{G}$ satisfying $\pi \mid \Gamma=\operatorname{Id}_{V_{\pi}}$.

So $\widehat{G / \Gamma}=\left\{\pi \in \hat{G} /\left.\pi\right|_{\Gamma}=\operatorname{Id}_{V_{n}}\right\} \subset \hat{G}$. We introduce the notations $\mathscr{C}(G / \Gamma)_{\pi}$, and $\mathscr{C}(G)_{\pi}$ as in (2.1).

(5.1) Lemma. The restriction of $\phi$ to $\mathscr{H}(G)^{\Gamma}$ is an isomorphism onto $\mathscr{H}(G / \Gamma)$. One has:

$$
\mathscr{H}(G)^{\Gamma}=\sum_{\pi \in \widehat{G} \widehat{\Gamma}} \mathscr{C}(G)_{\pi}
$$


Proof. Consider $\psi=\left.\phi^{-1}\right|_{\mathscr{H}(G / \Gamma)}$. It is clear that $\psi\left(\mathscr{C}(G / \Gamma)_{\pi}\right) \subset \mathscr{C}(G)_{\pi}$, if $\pi \in \widehat{G / \Gamma}$, and the Peter-Weyl theorem gives $\operatorname{dim} \mathscr{C}(G / \Gamma)_{\pi}=\operatorname{dim} \mathscr{C}(G)_{\pi}=(\operatorname{dim} \pi)^{2}$, if $\pi \in \widehat{G / \Gamma}$, so $\psi(\mathscr{C}(G / \Gamma))=\sum_{\pi \in G / \Gamma} \mathscr{C}(G)_{\pi}$. Now $\mathscr{H}(G)^{\Gamma}$ is a sub $G$-module of $\mathscr{H}$ for the left regular representation $L$, so it reduces on isotypical components:

$$
\mathscr{H}(G)^{\Gamma}=\sum_{\pi \in \hat{G}^{\prime}} \mathscr{H}(G)^{\Gamma} \cap \mathscr{C}(G)_{\pi} .
$$

Given $f \in \mathscr{H}(G)^{\Gamma}, \gamma \in \Gamma$, one has $L_{\gamma}(f)=f$ since $\Gamma$ is normal, so $\left.L_{\gamma}\right|_{\mathscr{H}(G)^{r} \cap \mathscr{C}(G)_{\pi}}=$ Id. But $L$ acts on $\mathscr{C}(G)_{\pi}$ as a sum of $\operatorname{dim} \pi$ representations isomorphic to $\check{\pi}$, so there are two cases: $\mathscr{H}(G)^{\Gamma} \cap \mathscr{C}(G)_{\pi} \neq\{0\}$, and then $\pi \in \widehat{G / \Gamma}$, or $\mathscr{H}(G) \cap \mathscr{C}(G)_{\pi}=\{0\}$. When $\pi \in \widehat{G / \Gamma}$, any element of $\mathscr{C}(G)_{\pi}$ is in $\mathscr{H}(G)^{\Gamma}$ ( $\Gamma$ is normal), so we conclude that

$$
\mathscr{H}(G)^{\Gamma}=\sum_{\pi \in G / \Gamma} \mathscr{C}(G)_{\pi}=\psi(\mathscr{H}(G / \Gamma)) .
$$

Now, the coproduct of $\mathscr{H}(G / \Gamma)$ induces a coproduct on $\mathscr{H}(G)^{\Gamma}$, and it is easy to check on coefficients (see (2.4.1)) that it is exactly the restriction of the coproduct of $\mathscr{H}(G)$. So $\mathscr{H}(G / \Gamma) \simeq \mathscr{H}(G)^{\Gamma}$ is a Hopf subalgebra of $\mathscr{H}(G)$.

(5.2) Proposition. Let $\tilde{\times}$ be a preferred deformation of the product of $\mathscr{H}(G)$ (resp.: $H(G)$ ), then $\tilde{x}$ can be restricted to $\mathscr{H}(G)^{\Gamma}$ (resp: $H(G)^{\Gamma}$ ), i.e.:

$\forall h, h^{\prime} \in \mathscr{H}(G)^{\Gamma}$ (resp: $\left.H(G)^{\Gamma}\right), \quad h \tilde{\times} h^{\prime} \in \mathscr{H}(G)^{\Gamma}[[t]]$ (resp: $\left.H(G)^{\Gamma}[[t]]\right)$, and defines a preferred deformation of $\mathscr{H}(G)^{\Gamma}$ (resp.: $\left.H(G)^{\Gamma}\right)$.

Proof. We begin by $\mathscr{H}(G)^{\Gamma}$. We can restrict to $h=C_{M}^{\pi}, h^{\prime}=C_{M^{\prime}}^{\pi^{\prime}}, \pi, \pi^{\prime} \in \widehat{G / \Gamma}$, $M \in \mathscr{L}\left(V_{\pi}\right), M^{\prime} \in \mathscr{L}\left(V_{\pi^{\prime}}\right)$.

By (4.3.2):

$$
C_{M}^{\pi} \tilde{\times} C_{M^{\prime}}^{\pi^{\prime}}=C_{M \otimes M^{\prime}}^{\pi \tilde{\otimes} \pi^{\prime}}
$$

But $\pi \tilde{\otimes} \pi^{\prime}=\tilde{P} \pi \otimes \pi^{\prime} \tilde{P}^{-1}$, with $\tilde{P} \in \mathscr{L}\left(V_{\pi} \otimes V_{\pi^{\prime}}\right)[[t]]$ (4.2.4).

So $C_{M}^{\pi} \tilde{\times} C_{M^{\prime}}^{\pi^{\prime}}=C_{\tilde{\boldsymbol{P}}-1}^{\pi{ }^{\prime} \pi_{M}^{\prime} \otimes M^{\prime}} \tilde{\boldsymbol{P}}$. Using (4.1.2), we compute:

$$
\begin{aligned}
x \in G, \gamma \in \Gamma, C_{M}^{\pi} \tilde{x} C_{M^{\prime}}^{\pi^{\prime}}(x \gamma) & =\tilde{\operatorname{Tr}}\left(\tilde{P}^{-1} \cdot M \otimes M^{\prime} \cdot \tilde{P} \cdot \pi \otimes \pi^{\prime}(x \gamma)\right) \\
& =\tilde{\operatorname{Tr}}\left(\tilde{P}^{-1} \cdot M \otimes M^{\prime} \cdot \tilde{P} \cdot \pi \otimes \pi^{\prime}(x)\right) \text { since } \pi \otimes \pi^{\prime} \in \widehat{G / \Gamma} \\
& =C_{M}^{\pi} \tilde{\times} C_{M^{\prime}}^{\pi^{\prime}}(x),
\end{aligned}
$$

which proves that $C_{M}^{\pi} \tilde{\times} C_{M^{\prime}}^{\pi^{\prime}} \in \mathscr{H}(G)^{\Gamma}[[t]]$, as wanted.

Now, we use $\overline{\mathscr{H}(G / \Gamma)}=H(G / \Gamma)$ to deduce $\overline{\mathscr{H}(G)^{\Gamma}}=H(G)^{\Gamma}$.

We write, for $h, h^{\prime} \in H(G)$ :

$$
h \tilde{\times} h^{\prime}=\sum_{n} t^{n} C_{n}\left(h, h^{\prime}\right) .
$$

By (4.3.4), $C_{n}\left(h, h^{\prime}\right) \in \mathscr{H}(G)$ if $h, h^{\prime} \in \mathscr{H}(G)$, by the beginning of the proof, $C_{n}\left(h, h^{\prime}\right) \in \mathscr{H}(G)^{\Gamma}$ if $h, h^{\prime} \in \mathscr{H}(G)^{\Gamma}$.

Given $h, h^{\prime} \in H(G)^{\Gamma}$, we choose sequences $\left(h_{p}\right),\left(h_{p}^{\prime}\right) \in \mathscr{H}(G)^{\Gamma}$ such that $\lim _{p} h_{p}=h$ and $\lim _{p} h_{p}^{\prime}=h^{\prime}$. From the continuity of $C_{n}$, the sequence $C_{n}\left(h_{p}, h_{p}^{\prime}\right)$ converges in $H(G)$ to $C_{n}\left(h, h^{\prime}\right)$, but since $C_{n}\left(h_{p}, h_{p}^{\prime}\right) \in \mathscr{H}(G)^{\Gamma} \subset H(G)^{\Gamma}, \forall p$, and since $H(G)^{\Gamma}$ is closed in $H(G)$, it results that $C_{n}\left(h, h^{\prime}\right) \in H(G)^{\Gamma}, \forall n$, and, therefore, that $h \tilde{\times} h^{\prime} \in H(G)^{\Gamma}[[t]]$.

So any preferred deformation of $H(G)$ (resp: $\mathscr{H}(G)$ ) restricts to $H(G)^{\Gamma}$ (resp: $\left.\mathscr{H}(G)^{\Gamma}\right)$; the obtained deformation of $H(G)^{\Gamma}$ (resp: $\left.\mathscr{H}(G)^{\Gamma}\right)$ is a preferred deformation because the coproduct of $H(G)^{\Gamma}$ (resp: $\left.\mathscr{H}(G)^{\Gamma}\right)$ is the restriction of the coproduct of $H(G)$ (resp: $\mathscr{H}(G)$ ), as mentioned above.

Q.E.D. 
(5.3) Remark. Using the isomorphism of Hopf algebras $\mathscr{H}(G)^{\Gamma} \simeq \mathscr{H}(G / \Gamma)$ (resp: $H(G)^{\Gamma} \simeq H(G / \Gamma)$ ) and (5.2), we see that any preferred deformation of $\mathscr{H}(G)$ (resp: $H(G)$ ) provides a preferred deformation of $\mathscr{H}(G / \Gamma)$ (resp: $H(G / \Gamma)$ ); this is the justification for the term quotient deformation. This result is useful, even if $G$ is simple (in which case $\Gamma$ is a finite subgroup of the center of $G$ ), because if one starts with a simple simply connected compact $G$ (this is exactly the general case of universal covering of a simple compact group), from deformations at the level of $G$, using (5.2), we get deformations at the level of any group covered by $G$ (e.g.: deformations for $S U(2)$ will provide deformations for $S O(3)$ ). One can even remove the condition that $\Gamma$ be normal by considering comodule algebras. However, if $\Gamma$ is not normal, there will be conditions on the deformed product of $\mathscr{H}$, or $H$, if one wants to get directly "a quantized homogeneous space" $G / \Gamma$. This will be treated elsewhere.

\section{Quantum Groups and Deformation Theory}

(6.1) Generators. It was mentioned in (2.4.2), (2.4.3) that $\mathscr{H}=\mathscr{H}(G)$ is a domain, and a finitely generated algebra. We need some facts about generators of $\mathscr{H}$, when $G$ is simple. They are classical, but since we don't know where they are completely explained, we give some details. We follow [4], Chap. VI.

(6.1.1) Definition. A subset $\left\{\pi_{1} \ldots \pi_{r}\right\}$ of $\hat{G}$ is said complete if the coefficients of $\pi_{1}, \ldots, \pi_{\mathrm{r}}$ provide a generator system of $\mathscr{H}$.

Using the Stone-Weierstrass and Peter-Weyl theorems, it is easy to check that $\left\{\pi_{1} \ldots \pi_{r}\right\}$ is complete if:

(1) $\forall i=1, \ldots, r, \check{\pi}_{i} \in\left\{\pi_{1}, \ldots, \pi_{r}\right\}$.

(2) $\pi=\bigoplus_{i=1}^{r} \pi_{i}$ is a faithful representation of $G$.

Faithful finite dimensional representations do exist for any compact connected Lie group, so complete sets do exist, and this proves that $\mathscr{H}$ is finitely generated.

(6.1.2) Proposition. (1) If $G=S U(n)$, or $S O(n)$, or $S p(n)$, and $\pi_{s}$ is the standard natural representation, then $\left\{\pi_{s}\right\}$ is a complete set.

(2) If $G=\operatorname{Spin}(n)$, there are two cases:

(i) $n=2 p+1$, the irreducible spin representation [4] is a complete set.

(ii) $n=2 p, \pi_{ \pm}$the two irreducible spin representations [4], then $\left\{\pi_{+}, \pi_{-}\right\}$is a complete set.

(3) We denote by $\tilde{G}_{2}, \tilde{F}_{4}, \tilde{E}_{6}, \tilde{E}_{7}, \tilde{E}_{8}$ the compact simply connected Lie groups with respective Lie algebra $g_{2}, f_{4}, e_{6}, e_{7}$ and $e_{8}$, then:

(i) If $G=\tilde{G}_{2}, \tilde{F}_{4}$ or $\tilde{E}_{8}$, any irreducible f.d. representation is a complete set.

(ii) If $G=\tilde{E}_{7}$, there exists an irreducible f.d. representation which is a complete set.

(iii) If $G=\tilde{E}_{6}$, there exist two irreducible f.d. representations such that $\left\{\pi_{1}, \pi_{2}\right\}$ is a complete set.

Proof. In case (1), if $G=S O(n)$ or $S p(n), \pi_{s}$ is faithful and self contragredient, so we apply the above criteria. If $G=S U(n), \check{\pi}_{s}=\operatorname{Ext}_{n-1}\left(\pi_{s}\right)$, so (1) is true. 
In case (2) (i), the irreducible spin representation is faithful and self contragredient; in case (2) (ii), $\left\{\pi_{+}, \pi_{-}\right\}$satisfies the conditions of the criteria.

For exceptional $\widetilde{G}_{2}, \widetilde{F}_{4}$ or $\widetilde{E}_{8}$, the center $Z(G)$ is trivial [15], and any irreducible f.d. representation is self contragredient [20], therefore (3) (i) is true.

For $\tilde{E}_{7}$, the center $Z(G)$ is $Z_{2}$ [15], any f.d. irreducible is self contragredient [20], so any irreducible subrepresentation of $\operatorname{Ind}_{Z(G)}^{G} \varepsilon, \varepsilon$ the alternate character of $Z_{2}$, will provide a complete set.

For $\tilde{E}_{6}$ the center is $Z_{3}[15]$ and we can find faithful irreducible representations by reduction of $\operatorname{Ind}_{Z(G)}^{G} \xi$, where $\xi$ is a faithful character of $Z(G)$; given such a $\pi$, then $\{\pi, \check{\pi}\}$ is a complete set; in that case, $\check{\pi} \neq \pi$ if $\pi$ is faithful, because $Z(G)=Z_{3}$.

Q.E.D.

Generator systems will provide a description of deformations of $\mathscr{H}$ which is quite similar to the FRT-model of quantum groups:

(6.1.3) Proposition. Assume that $G$ is one of the groups listed in (6.1.2). Let $\pi_{0}$ be the direct sum of the irreducible f.d. representations of $G$ appearing in a complete set as described in (6.1.2) and $\left\{C_{i j}\right\}$ the coefficients of $\pi_{0}$ in a fixed basis. If $\tilde{x}$ is a preferred Hopf deformation of $\mathscr{H}$, then $\left\{C_{i j}\right\}$ is a (topological) generator system of the $\mathbb{C}[[t]]$-algebra $(\mathscr{H}[[t]], \tilde{x})$; if $T$ is the matrix $\left[C_{i j}\right], T_{1}=T \otimes \mathrm{Id}, T_{2}=\mathrm{Id} \otimes T$, then there exists an invertible $\mathscr{R}$ in $\mathscr{L}\left(V_{\pi_{0}} \otimes V_{\pi_{0}}\right)[[t]]$ such that:

$$
\mathscr{R}\left(T_{1} \tilde{\times} T_{2}\right)=\left(T_{2} \tilde{\times} T_{1}\right) \mathscr{R}
$$

(here, by topological generator system we mean that the closure of the $\mathbb{C}[[t]]$ algebra generated by $\left\{C_{i j}\right\}$ is $\mathscr{H}[[t]]$ ).

Proof. First, we note that formula (4.3.2) can easily be generalized to any formal representations $\tilde{\pi}$ and $\tilde{\pi}^{\prime}$ of $G$, which are deformations of f.d. representations $\pi$ and $\pi^{\prime}(\operatorname{see}(4.1 .1))$ :

(6.1.4) $C_{M}^{\tilde{\pi}} \tilde{\times} C_{M^{\prime}}^{\tilde{\pi}^{\prime}}=C_{M \otimes M^{\prime}}^{\tilde{\pi} \tilde{\otimes} \tilde{\pi}^{\prime}}$, if $M \in \mathscr{L}\left(V_{\pi}\right), M^{\prime} \in \mathscr{L}\left(V_{\pi^{\prime}}\right)$, (see (4.1.2) for the definition of generalized coefficients). From the associativity of $\tilde{x}$, the new tensor product of representations of $G$ is associative, so we deduce:

$C_{M_{1}}^{\tilde{\pi}_{1}} \tilde{\times} \ldots \tilde{\times} C_{M_{n}}^{\tilde{\pi}_{n}}=C_{M_{1} \otimes}^{\tilde{\pi}_{1} \tilde{\otimes}} \stackrel{\tilde{\otimes} \tilde{\pi}_{n}}{\otimes}$, if $\tilde{\pi}_{i}$ deforms f.d. $\pi_{i}$, and $M_{i} \in \mathscr{L}\left(V_{\pi}\right), i=1 \ldots n$.

Now we take $\tilde{\pi}_{i}=\pi_{0}, \forall i$ and set $\pi_{0} \tilde{\otimes} \cdots \tilde{\otimes} \pi_{0}=\tilde{\otimes}_{n} \pi_{0}, M_{e}=e_{i_{e}}^{*} \otimes e_{j_{e}},\left\{e_{i}\right\}$ being a basis of $V_{\pi_{0}}$, and obtain:

$$
C_{i_{1} j_{1}} \tilde{\times} \cdots \tilde{\times} C_{i_{n} j_{n}}=C_{M}^{\tilde{\otimes}_{n} \pi_{0}}, \quad \text { with } M=\left(e_{i_{1}}^{*} \otimes \cdots \otimes e_{i_{n}}^{*}\right) \otimes\left(e_{j_{1}} \otimes \cdots \otimes e_{j_{n}}\right) .
$$

Since $\tilde{\otimes}_{n} \pi_{0}$ is a deformation of $\otimes_{n} \pi_{0}$, it results from (4.1.4) that we can find $\tilde{P} \in \mathscr{L}\left(\otimes_{n} V_{\pi_{0}}\right)[[t]]$ such that $\widetilde{\otimes}_{n} \pi_{0}=\tilde{P} \cdot \otimes_{n} \pi_{0} \cdot \tilde{P}^{-1}$. Then, by (2.3) and (4.1.2):

$$
C_{i_{1} j_{1}} \times \ldots \times C_{i_{n} j_{n}}=C_{M}^{\otimes_{n} \pi_{0}}=C_{\tilde{P} M \tilde{P}-1}^{\tilde{\otimes}_{n} \pi_{0}}
$$

But $\quad \tilde{P} M \tilde{P}^{-1}=\sum_{\alpha_{1}, \alpha_{n}, \beta_{1}} \quad{ }_{\beta_{n}} \tilde{\lambda}_{\alpha_{1}} \quad \alpha_{n} \beta_{1} \quad \beta_{n}\left(e_{\alpha_{1}}^{*} \otimes \cdots \otimes e_{\alpha_{n}}^{*}\right) \otimes\left(e_{\beta_{1}} \otimes \cdots \otimes e_{\beta_{n}}\right)$, with $\tilde{\lambda}_{\alpha_{1}} \alpha_{n} \beta_{1} \quad \beta_{n} \in \mathbb{C}\left[\dot{\alpha_{1}}[t]\right]$, so:

$$
C_{i_{1} j_{1}} \times \cdots \times C_{i_{n} j_{n}}=\sum_{\alpha_{1}} \sum_{\alpha_{n}, \beta_{1}} \tilde{\lambda}_{\alpha_{1}} \alpha_{n} \beta_{1}, \beta_{n} C_{\alpha_{1} \beta_{1}} \tilde{\times} C_{\alpha_{2} \beta_{2}} \tilde{\times} \cdots \tilde{\times} C_{\alpha_{n} \beta_{n}} .
$$

Therefore any polynomial in the $\left\{C_{i j}\right\}$ for the initial product can be written as a polynomial of $\left\{C_{i j}\right\}$ for the new product. Note that the degree does not increase, and that, in the new product case, we are dealing with non-commutative 
polynomials with coefficients in $\mathbb{C}[[t]]$. Now any $h$ in $\mathscr{H}[[t]]$ can be written $h=\sum_{n} t^{n} h_{n}, h_{n} \in \mathscr{H}$, and any $h_{n}$ is a non-commutative $\mathbb{C}[[t]]$-polynomial in $\left\{C_{i j}\right\}$ for the new product, so $\left\{C_{i j}\right\}$ is a topological generator system of $(\mathscr{H}[[t]], \tilde{x})$.

As to relation $\mathscr{R} \cdot T_{1} \tilde{\times} T_{2}=T_{1} \tilde{\times} T_{2} \cdot \mathscr{R}$, it is an equivalent (but illuminating) way to express the quasicommutativity (4.3.1) of $\tilde{x}$, combined with formula (4.3.2), as we now show:

Let $\tilde{\Delta^{\prime}}=\sigma \cdot \tilde{\Delta}$ and denote by $\tilde{\rho}$ the new tensor product of $\pi_{0}$ by itself obtained from $\tilde{\Delta}$, and by $\tilde{\rho}^{\prime}$ the new tensor product of $\pi_{0}$ by itself obtained from $\tilde{\Delta}^{\prime}$. Then, by the quasicocommutativity of $\tilde{\Delta}(4.2 .5)$, one has $\widetilde{\Delta}^{\prime}=\tilde{\mathscr{R}} \cdot \tilde{\Delta} \cdot \tilde{\mathscr{R}}^{-1}$, so if we set $\mathscr{R}=\pi_{0} \otimes \pi_{0}(\tilde{\mathscr{R}})$, we get that $\tilde{\rho}^{\prime}=\mathscr{R} \tilde{\rho} \mathscr{R}^{-1}$. Using now (6.1.4), the notation $E_{i j}=e_{i}^{*} \otimes e_{j}$, and ${ }^{T} \Delta^{\prime}\left(h, h^{\prime}\right)=h^{\prime} \tilde{\times} h$, we deduce:

$$
\begin{aligned}
& C_{i^{\prime} j^{\prime}} \tilde{\times} C_{i j}=C_{E_{i j} \otimes E_{i^{\prime} j^{\prime}}}^{\tilde{\rho}^{\prime}}=C_{E_{i j} \otimes E_{i^{\prime} j^{\prime}}}^{\mathscr{R} \tilde{\rho} \tilde{R}-1}=C_{\left(i, i^{\prime}\right)\left(j, j^{\prime}\right)}^{\mathscr{R} \tilde{\rho} \mathscr{R}-1} \text {, and } \\
& C_{i j} \tilde{\times} C_{i^{\prime} j^{\prime}}=C_{E_{i j} \otimes E_{i j^{\prime}}}^{\tilde{\rho}}=C_{\left(i, i^{\prime}\right)\left(j, j^{\prime}\right)}^{\tilde{\rho}},
\end{aligned}
$$

so that from these two relations, one has:

$$
T_{2} \tilde{\times} T_{1}=\mathscr{R}\left(T_{1} \tilde{\times} T_{2}\right) \mathscr{R}^{-1} .
$$

(6.1.5) Remark. $\left\{C_{i j}\right\}$ being a generator system of $(\mathscr{H}, \times), \mathscr{H}$ has a natural filtration coming from the one of the polynomial algebra $\mathbb{C}\left[C_{i j}\right]$. Now, let us define a topological filtration in a topological $\mathbb{C}[[t]]$-algebra to be an increasing sequence of $\mathbb{C}[[t]]$-submodules $A_{n}$ such that $A_{n} \cdot A_{p} \subset A_{n+p}, \forall n, p$ and $\overline{\bigcup_{n} A_{n}}=A$. Then $(\mathscr{H}[[t]], \times)$ has a topological filtration inherited from $\mathbb{C}\left[C_{i j}\right]$, as said above, and, from the proof of (6.1.3), this filtration also works for $(\mathscr{H}[[t]], \tilde{x})$. Moreover, from $\mathscr{R} T_{1} T_{2}=T_{2} T_{1} \mathscr{R}$, any monomial in $\left\{C_{i j}\right\}$ for the new product can be reordered, so that elements of degree at most $n$ are actually $\mathbb{C}[[t]]$-linear combinations of ordered monomials of degree at most $n$ in the new product (roughly speaking, the graded associated algebra is still $\mathbb{C}\left[C_{i j}\right]$ ).

(6.2) The Drinfeld Models. We give a brief description of the D-models of quantum groups, following [6,8]: Given a simple complex f.d. Lie algebra $g$, with $\mathscr{U}$ its enveloping algebra, there exists a Hopf deformation $\mathscr{U}_{t}$ of $\mathscr{U}$ which is a topologically free complete $\mathbb{C}[[t]]$-module (i.e. $\mathscr{U}_{t} \simeq \mathscr{U}[[t]]$ as $\mathbb{C}[[t]]$-modules), with coproduct $\tilde{\Delta}: \mathscr{U}_{t} \rightarrow \mathscr{U}_{t} \hat{\otimes}_{t} \mathscr{U}_{t}$; therefore $\mathscr{U}_{t}$ is a deformation of $\mathscr{U}$ in the sense of (3.7), when $\mathscr{U}$ is given its natural topology. It is shown in [8] that $\mathscr{U}$ is (deformation) rigid, therefore $\mathscr{U}_{t} \simeq \mathscr{U}[[t]]$ as an algebra, and that $\tilde{\Delta}$ is obtained from the standard coproduct $\Delta_{0}$ by a twist, i.e. there exists $\tilde{P} \in \mathscr{U}_{t} \hat{\otimes}_{t} \mathscr{U}_{t}=(\mathscr{U} \otimes \mathscr{U})[[t]]$ such that $\tilde{\Delta}=\tilde{P} \Delta_{0} \tilde{P}^{-1}$. Let us note the analogy between these results and (4.2.1), (4.2.4). Now, let $G$ be the compact simply connected Lie group with Lie algebra $g_{0}$ such that $g_{0} \otimes_{\mathbb{R}} \mathbb{C}=g$. We introduce $H=H(G)=C^{\infty}(G), \quad A=A(G)=H(G)^{*}$, $\mathscr{H}=\mathscr{H}(G)$ and $\mathscr{A}=\mathscr{A}(G)$ as in Sect. 2; using (2.6.2), $\mathscr{U} \subset A \subset \mathscr{A}$, so $\mathscr{U}[[t]] \subset A[[t]] \subset \mathscr{A}[[t]]$.

(6.2.1) Proposition. The Hopf deformation $\mathscr{U}_{t}$ can be extended to a Hopf deformation of $A$ (resp: $\mathscr{A})$ with unchanged product, unit and counit.

Using $\mathbb{C}[[t]]$-duality (3.8), we deduce:

(6.2.2) Corollary. The Hopf deformation $\mathscr{U}_{t}$ produces (by $\mathbb{C}[[t]]$-duality) a preferred Hopf deformation of $\mathscr{H}$ and $H$. 
(6.2.3) Remark. So our results, and especially (4.3.2), (4.3.3) and (6.1.3) can be applied. Note that the $\mathscr{R}$-matrix of (6.1.3) can be specified to be a solution of the Yang-Baxter equation, because in the D-model, the twist between $\tilde{\Delta}$ and $\sigma \cdot \tilde{\Delta}$ can be chosen with this property [6]. It is interesting that the D model will produce on $\mathscr{H}$ a preferred deformation for any $G$ listed in (6.1.2), and not only for $S U(n), S O(n)$ and $S p(n)$ as the FRT-model does (see also Remark 23 in [9]). In the case of $\operatorname{Spin}(2 p)$ and $\tilde{E}_{6}$, the coefficients of two (and not only one) irreducible f.d. representations will be needed to describe the product as in (6.1.3). We also note that nobody has ever found a preferred deformation on $H$, which is however, in our opinion, a very good candidate for deformation of Poisson brackets!

Proof of (6.2.1). Since $\tilde{\Delta}=\tilde{P} \Delta_{0} \tilde{P}^{-1}$, with $\tilde{P} \in(\mathscr{U} \otimes \mathscr{U})[[t]], \tilde{\Delta}$ extends to a coproduct on $A[[t]]$, and $\mathscr{A}[[t]]$. With this new coproduct and the standard product, $A[[t]]$ and $\mathscr{A}[[t]]$ are $\mathbb{C}[[t]]$-bialgebras. Using (3.10) there exists an antipode and a counit. From (4.2.6), this counit is the standard counit of $A$, or $\mathscr{A}$; since it restricts to $\mathscr{U}_{t}=\mathscr{U}[[t]]$, by unicity of the counit, the restriction has to be the counit of $\mathscr{U}_{t}$. Now let $\tilde{S}$ be the antipode of $\mathscr{U}_{t}$, then given any irreducible representation $\pi$ of $g$, we define a deformation $\tilde{\rho}={ }^{T} \pi \circ \tilde{S}$ of $\check{\pi}$. Since $g$ is simple, $\check{\pi}$ is rigid, so $\tilde{\rho}=\alpha_{\pi} \circ \check{\pi} \circ \alpha_{\pi}^{-1}$, with $\alpha_{\pi} \in \mathscr{L}\left(V_{\pi}^{*}\right)[[t]]$. But $\check{\pi}={ }^{T} \pi \circ S_{0}$, with $S_{0}$ the standard antipode of $\mathscr{A}$, so $\pi(\tilde{S}(u))=a_{\pi} \circ \pi\left(S_{0}(u)\right) \circ a_{\pi}^{-1}, \quad \forall u \in \mathscr{U}$, with $a_{\pi}={ }^{T} \alpha_{\pi}^{-1} \in \mathscr{L}\left(V_{\pi}\right)[[t]]$; so we can extend $\tilde{S}$ to $\mathscr{A}[[t]]$ by the following formula:

$$
b \in \mathscr{A}, \quad \tilde{S}(b)_{\pi}=a_{\pi} S_{0}(b)_{\pi} a_{\pi}^{-1}=a_{\pi}^{T} b_{\check{\pi}} a_{\pi}^{-1} .
$$

This defines a second antipode for the above mentioned Hopf structure of $\mathscr{A}[[t]]$; by unicity of the antipode, it must be the same.

By (3.10), $A[[t]]$, with its coproduct $\tilde{\Delta}$, has an antipode $\tilde{S^{\prime}}$, and by (4.2.6), one has $\tilde{S^{\prime}}=\tilde{a} S_{0} \tilde{a}^{-1}$, with $\tilde{a} \in A[[t]]$. But this last formula defines an antipode on $\mathscr{A}[[t]]$ with its coproduct $\tilde{\Delta}$, so by unicity of the antipode, $\tilde{S}^{\prime}=\tilde{S}$. So we have proved that the coproduct, counit and antipode of $\mathscr{U}_{t}=\mathscr{U}[[t]]$ extend to $A[[t]]$ and $\mathscr{A}[[t]]$.

Q.E.D.

(6.3) Drinfeld Isomorphisms. We continue with the notations of (6.2) and discuss the following problem: in (6.2.1) an isomorphism $\tilde{\varphi}: \mathscr{U}_{t} \simeq \mathscr{U}[[t]]$ (called Drinfeld isomorphism in the following) is fixed. Now, such a Drinfeld isomorphism is certainly not unique; what happens if it is changed?

(6.3.1) Proposition. Let $\tilde{\varphi}$ and $\tilde{\psi}$ two Drinfeld isomorphisms, then the corresponding preferred deformations of $\mathscr{H}(G)$ are equivalent.

Before proving (6.3.1), let us note that a good choice of the Drinfeld isomorphism has still some importance, because it will simplify defining relations of the corresponding deformation of $\mathscr{H}(G)$. We shall come back to this problem in next subsections.

Proof of (6.3.1). Drinfeld isomorphisms are constructed as follows: take any section of the canonical morphism $\mathscr{U}_{t} \rightarrow \mathscr{U}$ (such sections do exist by rigidity of $\mathscr{U}$, see $[8])$, and extend it to $\mathscr{U}[[t]]$ by $\mathbb{C}[[t]]$-linearity. Therefore, given $\tilde{\varphi}$ and $\tilde{\psi}$, and defining $\tilde{\theta}=\tilde{\psi} \circ \tilde{\varphi}^{-1}, \tilde{\theta}$ is a $\mathbb{C}[[t]]$-linear automorphism of $\mathscr{U}[[t]]$, and $\tilde{\theta}_{0}=\operatorname{Id}_{\mathscr{U}}$. It results that $\pi \circ \tilde{\theta}, \pi \in \hat{G}$, is a deformation of the representation $\pi$ of $g$, and since $g$ is 
simple, $\pi \circ \tilde{\theta}=\tilde{\alpha}_{\pi} \circ \pi \circ \tilde{\alpha}_{\pi}^{-1}$, with $\alpha_{\pi} \in \mathscr{L}\left(V_{\pi}\right)[[t]]$. Now let $\tilde{a}=\left(\tilde{\alpha}_{\pi}\right) \in \mathscr{A}[[t]]=$ $\prod_{\pi \in \hat{G}} \mathscr{L}\left(V_{\pi}\right)[[t]]$; we extend $\tilde{\theta}$ to (a continuous automorphism of ) $\mathscr{A}[[t]]$ by:

$$
\tilde{\theta}(\tilde{b})=\tilde{a} \tilde{b} \tilde{a}^{-1}, \quad \tilde{b} \in \mathscr{A}[[t]] .
$$

The coproduct $\tilde{\Delta}$ of $\mathscr{U}_{t}$ induces coproducts $\tilde{\Delta}_{\varphi}$ and $\tilde{\Delta}_{\psi}$ of $\mathscr{U}[[t]]$ by formula:

$$
\tilde{\Delta}_{\varphi}=\tilde{\varphi} \otimes \tilde{\varphi} \circ \tilde{\Delta} \circ \tilde{\varphi}^{-1} \quad\left(\operatorname{resp}: \tilde{\Delta}_{\psi}=\tilde{\psi} \otimes \tilde{\psi} \circ \tilde{\Delta} \circ \tilde{\psi}^{-1}\right) \text {. }
$$

Now, one has $\tilde{\Delta}_{\psi}=\tilde{\theta} \otimes \tilde{\theta} \circ \tilde{\Delta}_{\varphi} \circ \tilde{\theta}^{-1}$. It was shown in (6.2.1) that $\tilde{\Delta}_{\varphi}$ and $\tilde{\Delta}_{\psi}$ extend to (continuous) coproducts on $\mathscr{A}[[t]]$. Since $\tilde{\theta}$ extends to $\mathscr{A}[[t]]$, and since $\overline{\mathscr{U}[[t]]}=\mathscr{A}[[t]](2.6 .1)$, the formula $\tilde{\Delta}_{\psi}=\tilde{\theta} \otimes \tilde{\theta} \circ \tilde{\Delta}_{\varphi} \circ \tilde{\theta}^{-1}$ is valid for the extensions of $\tilde{\Delta}_{\varphi}$ and $\tilde{\Delta}_{\psi}$ to $\mathscr{A}[[t]]$. So $\left(\mathscr{A}[[t]], \tilde{\Delta}_{\varphi}\right)$ and $\left(\mathscr{A}[[t]], \tilde{\Delta}_{\psi}\right)$ are equivalent deformations of $\left(\mathscr{A}, \Delta_{0}\right)$, and (3.8) finishes the proof of $(6.3 .1)$.

Q.E.D.

(6.4) FRT-Models. We now show how FRT-models are recovered by a good choice of the Drinfeld isomorphism. First, we need the following lemma:

(6.4.1) Lemma. Let $\tilde{\rho}$ be a representation of $\mathscr{U}_{t}$, and $\pi=\tilde{\rho}_{0}, \pi \in \hat{G}$. Then there exists a Drinfeld isomorphism $\tilde{\varphi}$ such that $\tilde{\rho}=\pi \circ \tilde{\varphi}$.

Proof. Fix any Drinfeld isomorphism $\tilde{\varphi}^{\prime}$. Now $\tilde{\rho} \circ \tilde{\varphi}^{\prime-1}$ is a deformation of the representation $\pi$ of $g$, hence a trivial deformation. So there exists $\tilde{\alpha}_{\pi} \in \mathscr{L}\left(V_{\pi}\right)[[t]]$, such that $\tilde{\rho} \circ \tilde{\varphi}^{\prime-1}=\tilde{\alpha}_{\pi} \circ \pi \circ \tilde{\alpha}_{\pi}^{-1}$. We write $\tilde{\alpha}_{\pi}=\sum_{n} t^{n} \beta_{n}, \beta_{n} \in \mathscr{L}\left(V_{\pi}\right)$, and use the Jacobson density theorem: there exists $u_{n} \in \mathscr{U}$ such that $\beta_{n}=\pi\left(u_{n}\right)$, and therefore there exists $\tilde{u}=\sum_{n} t^{n} u_{n} \in \mathscr{U}[[t]]$ such that $\tilde{\alpha}_{\pi}=\pi(\tilde{u})$. Now we obtain that

$$
\begin{aligned}
\tilde{\rho} \circ \tilde{\varphi}^{\prime-1}(\tilde{b}) & =\pi\left(\tilde{u} \tilde{b} \tilde{u}^{-1}\right), \quad \forall \tilde{b} \in \mathscr{U}[[t]], \text { so: } \\
\tilde{\rho}(\tilde{c}) & =\pi\left(\tilde{u} \tilde{\varphi}^{\prime}(\tilde{c}) \tilde{u}^{-1}\right), \quad \forall \tilde{c} \in \mathscr{U}_{t} .
\end{aligned}
$$

We define a Drinfeld isomorphism $\tilde{\varphi}$ by $\tilde{\varphi}(\tilde{c})=\tilde{u} \tilde{\varphi}^{\prime}(\tilde{c}) \tilde{u}^{-1}, \forall \tilde{c} \in \mathscr{U}_{t}$, and then $\tilde{\rho}=\pi \circ \tilde{\varphi}$.

Q.E.D.

(6.4.2). Let us apply (6.4.1) to the case $G=S U(n)$. We follow closely ([6], Sect. 7), except that we replace $h$ by $t$, and we denote by $\tilde{\rho}$ the representation of $\mathscr{U}_{t}$ denoted by $\rho$ in that paper. Then $\tilde{\rho}(0)=\pi_{s}$, the standard natural representation of $g=s l(n)$, which is also the standard natural representation of $G$. We choose a Drinfeld isomorphism $\tilde{\varphi}$ such that $\tilde{\rho}=\pi_{s} \circ \tilde{\varphi}(6.4 .1)$. We denote by $C_{i j}$ the coefficients of $\pi_{s}$; they are a generator system of $\mathscr{H}$. The deformation $\mathscr{U}_{t}$ (and the choice of $\tilde{\varphi}$ ) produces a deformation of $\mathscr{H}$ (and $H$ ), and we want to compute the new relations of the generator system $\left\{C_{i j}\right\}$ (see (6.1.3)). But this computation is explicitly done in ([6], Sect. 7): let $\rho_{i j}$ be the coefficients of $\tilde{\rho}$, they are elements of $\left(\mathscr{U}_{t}\right)_{t}^{*}$; by the choice of $\tilde{\varphi}$, one has ${ }^{T} \tilde{\varphi}\left(C_{i j}\right)=\rho_{i j}$; the Hopf algebras $\left(\mathscr{U}_{t}, \tilde{\Delta}\right)$ and $\left(\tilde{U}[[t]], \tilde{\Delta}_{\tilde{\varphi}}=\right.$ $\left.\tilde{\varphi} \otimes \tilde{\varphi} \circ \tilde{\Delta} \circ \tilde{\varphi}^{-1}\right)$ are isomorphic by $\tilde{\varphi}$, so $\left(\mathscr{U}_{t}\right)_{t}^{*} \simeq(\mathscr{U}[[t]])_{t}^{*}=\mathscr{U}^{*}[[t]]$ by ${ }^{T} \tilde{\varphi}$. So in formulas (16), (17), (18), (19) given in ([6], Sect. 7) for the products of $\rho_{i j}$, one has only to replace $\rho_{i j}$ by $C_{i j}$ to get the relations between the generators $\left\{C_{i j}\right\}$ of $\mathscr{H}$ (or $H$ ), for the deformed product $\tilde{x}$ provided by (6.2.2). A glance at [9] is enough to be convinced that we recover (a formal version of) the FRT-quantization of $S L(n)$. Note that $(\mathscr{H}, \tilde{x})$ has an antipode (6.2.2), and so does the FRT-quantization; by unicity, they have to be the same. Actually, this proves that the FRT-quantization of $S L(n)$ can be seen as a preferred Hopf deformation of $\mathscr{H}(S U(n))$; this result has been obtained in [11], [12], by different techniques. Moreover, applying (6.2.2), this 
deformation extends to a preferred Hopf deformation of $H(G)=C^{\infty}(G)$, a result which is completely new.

(6.4.3) FRT-quantizations (or rather formal versions) can be similarly recovered from D-models in cases $G=S O(n)$, or $G=S p(n)$, and this proves that there exists a preferred Hopf deformation of $\mathscr{H}(S O(n))$ or $\mathscr{H}(S p(n))$ which satisfies the relations of FRT-quantizations as given in [9]. We insist that this result is a justification of the terminology "deformation," often employed, but never justified in these cases (see e.g. [11], where it is shown that relations of type $\mathscr{R} T_{1} T_{2}=T_{2} T_{1} \mathscr{R}$ need not define a deformation, even if $\mathscr{R}$ is Yang-Baxter). Now, the proof looks very much like the case of $S U(n)$, but cannot be so explicit, the main task being to choose the representation $\tilde{\rho}$ used above. This can be done using the reconstruction theorem 12 of [9], and the fundamental corepresentation $\tau$ defined in ([9], Def. 20). Applying the method developed in Sect. (6.4), the D-model will produce by (6.2.2) a preferred Hopf deformation of $\mathscr{H}(G)$ satisfying FRT-relations. Note that this deformation extends to a deformation of $H(G)=C^{\infty}(G)$.

(6.4.4). D-models and (6.2.2) predict the existence of a preferred Hopf deformation of $\mathscr{H}(G)$ (or $H(G)$ ) for any $G$ listed in (6.1.2), with generators $T=\left(C_{i j}\right)$ satisfying $T_{1} \tilde{\times} T_{2}=\mathscr{R} T_{2} \tilde{\times} T_{1} \mathscr{R}^{-1},(6.1 .3)$, and $\mathscr{R}$ Yang-Baxter (6.2.3). It would be of interest to describe such an FRT-model when e.g. $G=\operatorname{Spin}(n)$ and especially $\operatorname{Spin}(2 p)$, where two irreducible representations have to be used (6.1.2). Such a model will induce a preferred Hopf deformation of $\mathscr{H}(G / \Gamma)$, for any $\Gamma$ in the center of $G(5.3)$. The case of exceptional $G$ is also of interest, since very little seems to be known.

Finally, we mention that the Reshetikhin model (see [19], and also [22]), being obtained by twisting the standard coproduct, is also in our deformation framework: it will also produce deformations of $\mathscr{H}(G)$ (or $H(G)$ ) of the above type.

\section{J Models}

For complex $t \notin 2 \pi \mathbf{Q}$, we set $q=e^{i t}$, and define the J-model $A_{t}(g), g=s l(2)$, as the algebra generated by $\left\{F, G, K, K^{-1}\right\}$ with relations:

$$
[F, G]=\frac{K^{2}-K^{-2}}{q-q^{-1}}, \quad F K=q^{-1} K F, \quad G K=q K G .
$$

Direct interpretation in deformation theory is not possible, because (7.1) is not defined at $t=0$. Nevertheless, as shown in [5] or [3], it is not difficult to swallow this singularity, and actually define $A_{t}(g)$ for any $t \in \mathbb{C}$, by introducing $S=\frac{K-K^{-1}}{q-q^{-1}}, C=\frac{K+K^{-1}}{2}$, so that $A_{t}(g)$ is the algebra generated by $\{F, G, S, C\}$ with relations:

$$
\begin{gathered}
{[F, G]=2 S C, \quad F S=(S \cos t-C) F, \quad F C=\left(C \cos t+S \sin ^{2} t\right) F,} \\
G S=(S \cos t+C) G, \quad G C=\left(C \cos t-S \sin ^{2} t\right) G, \\
C^{2}+S^{2} \sin ^{2} t=1, \quad[S, C]=0,
\end{gathered}
$$


Though (7.2) is a rather lengthy definition, it does define $A_{t}(g)$ for any $t \in \mathbb{C}$. Moreover, if $t$ is now a formal parameter, we can define $\widetilde{A}_{t}(g)$ (the formal J-model) as the $\mathbb{C}[[t]]$-algebra generated by $\left\{S, C, K, K^{-1}\right\}$ with relations (7.2).

Now for $t=0,(7.2)$ becomes:

$$
\begin{gathered}
{[F, G]=2 S C, \quad[C, F]=[C, G]=[C, S]=0,} \\
{[S, F]=C F, \quad[S, G]=-C G, \quad C^{2}=1 .}
\end{gathered}
$$

Setting $\hat{Y}=S C, \hat{F}=F C, \hat{G}=G C$, one finds the commutation rules of $g=\operatorname{sl}(2)$. So $A_{0}(g) \simeq \mathscr{U}(g) \otimes P$, with $P \simeq \mathbb{C}[x] / x^{2}-1$, and this proves that the classical limit of the J-model is not $\mathscr{U}(g)$, as often asserted, but an extension of $\mathscr{U}(g)$ by a parity $C$. Let us note that a similar result holds for general simple $g$, by similar arguments: the classical limit of the J-model will be an extension of $\mathscr{U}(g)$ by $r$ parities $(r=\operatorname{rank} g)$. Now we come back to $g=s l(2)$. From the Poincaré-Birkhoff-Witt theorem, we deduce that $\tilde{A}_{t}(g)$ is a deformation of $A_{0}(g)$. It is well-known that $\tilde{A}_{t}(g)$ is a domain, and it is obvious that $A_{0}(g)$ is not, so the $\mathbb{C}[[t]]$-algebras $\tilde{A}_{t}(g)$ and $A_{0}(g)[[t]]$ cannot be isomorphic, which proves:

(7.3) Proposition. $A_{t}(g)$ is a non-trivial deformation of $A_{0}(g) \simeq \mathscr{U}(g) \otimes \mathbb{C}[x] / x^{2}-1$.

The same argument shows that the algebras $A_{t}(g)$ and $A_{0}(g)$ cannot be isomorphic when $t \notin 2 \pi \mathbf{Q}$. It was shown in [3] that $A_{t}(g) \simeq A_{t^{\prime}}(g)$ if and only if $t^{\prime}= \pm t+2 k \pi, k \in \mathbf{Z}$, and this has an intuitive interpretation as non-rigidity of $A_{t}(g)$ (and not only of $A_{0}(g)$ as shown in (7.3)). Let us now give a complete justification of this interpretation:

We fix $t_{0} \in \mathbb{C}$, and define $\widetilde{B}_{t}(g)$ as the $\mathbb{C}[[t]]$-algebra generated by $\{F, G, S, C\}$ with relations:

$$
\begin{gathered}
{[F, G]=2 S C, F S=\left(S \cos \left(t_{0}+t\right)-C\right) F, F C=\left(C \cos \left(t_{0}+t\right)+S \sin ^{2}\left(t_{0}+t\right) F,\right.} \\
G S=\left(S \cos \left(t_{0}+t\right)+C\right) G, \quad G C=\left(C \cos \left(t_{0}+t\right)-S \sin ^{2} t\right) G \\
C^{2}+S^{2} \sin ^{2}\left(t_{0}+t\right)=1, \quad[S, C]=0 .
\end{gathered}
$$

The classical limit $t=0$ is $A_{t_{0}}(g)$, and using once more the Poincaré-Birkhoff-Witt theorem, $\tilde{B}_{t}(g)$ is a deformation of $A_{t_{0}}(g)$.

We denote by $D_{n}$ the irreducible representation of $g$ of dimension $(2 n+1), n \in \frac{1}{2} \mathbf{N}$, and also by $D_{n}$ its extension to $A_{t_{0}}(g), t_{0} \notin 2 \pi \mathbf{Q}$, as defined in [3].

(7.5) Lemma. Let $(\pi, W)$ be a finite dimensional representation of $A_{t_{0}}(g), t_{0} \notin 2 \pi \mathbf{Q}$; then

$$
H^{1}\left(A_{t_{0}}(g), \mathscr{L}(W)\right)=\{0\} .
$$

(7.5.1) Corollary. When $t_{0} \notin 2 \pi \mathbf{Q}$, the finite dimensional representations of $A_{t_{0}}(g)$ are rigid.

Proof. Given $\xi \in Z^{1}\left(A_{t_{0}}(g), \mathscr{L}(W)\right)$, we define a new representation $\rho$ of $A_{t_{0}}(g)$ on $W \times W$ by $\rho=\left(\begin{array}{ll}\pi & \xi \\ 0 & \pi\end{array}\right)$. But $\rho$ is semi-simple, and since it is an extension of $\pi$ by itself [18], the extension cocycle $\xi$ is a coboundary. Now $H^{1}\left(A_{t_{0}}(g), \mathscr{L}(W)\right)=\{0\}$ is the standard sufficient condition for rigidity of $\pi$ [17].

Q.E.D.

(7.5.2) Proposition. When $t_{0} \notin 2 \pi \mathbf{Q}, \tilde{B}_{t}(g)$ is a non-trivial deformation of $A_{t_{0}}(g)$. 
(7.5.3) Corollary.

$$
H^{2}\left(A_{t_{0}}(g), A_{t_{0}}(g)\right) \neq\{0\}
$$

Proof. Let us assume that the $\mathbb{C}[[t]]$-algebras $A_{t_{0}}(g)[[t]]$ and $\tilde{B}_{t}(g)$ are isomorphic by $\phi$. Let $\tilde{\pi}_{i}$ be the $(2 i+1) \mathbb{C}[[t]]$-dimensional representation of $\tilde{B}_{t}(g)$ as defined in [3] (p. 79). Then the value of the Casimir element $Q_{t}=G F+S C+$ $S^{2} \cos \left(t_{0}+t\right)$ of $\tilde{B}_{t}(g)$ is $\sin i\left(t_{0}+t\right) \sin (i+1)\left(t_{0}+t\right) / \sin ^{2}\left(t_{0}+t\right)$.

At $t=0, B_{t}(g)$ has classical limit $A_{t_{0}}(g)$, and $\tilde{\pi}_{i}$ has classical limit $\pi_{i}$, the irreducible $(2 i+1)$-dimensional representation $\pi_{i}$ of $A_{t_{0}}(g)$. Then setting $\hat{\pi}_{i}=\tilde{\pi}_{i} \circ \phi$, we get a deformation of the representation $\pi_{i}$ of $A_{t_{0}}(g)$, which has to be trivial by (7.5.1), so the value of the Casimir $Q_{0}$ of $A_{t_{0}}(g)$ in $\hat{\pi}_{i}$ is $\sin i t_{0} \sin (i+1) t_{0} / \sin ^{2} t_{0}$.

Now, the respective centers of $\tilde{B}_{t}(g)$ and $A_{t_{0}}(g)[[t]]$ are $Z\left(\tilde{B}_{t}(g)\right)=\mathbb{C}[[t]]\left[Q_{t}\right]$, and $Z\left(A_{t_{0}}(g)[[t]]\right)=\mathbb{C}[[t]]\left[Q_{0}\right]$. One has $Z\left(B_{t_{0}}(g)\right)=\phi\left(Z\left(A_{t_{0}}(g)[[t]]\right)\right)$ $=\mathbb{C}[[t]]\left[\phi\left(Q_{0}\right)\right]$, therefore $\phi\left(Q_{0}\right)=\tilde{\alpha} Q_{t}+\tilde{\beta}$, with $\tilde{\alpha}, \tilde{\beta} \in \mathbb{C}[[t]]$, and $\hat{\pi}_{i}\left(Q_{0}\right)$ $=\tilde{\alpha} \tilde{\pi}_{i}\left(Q_{t}\right)+\tilde{\beta}(7.6 .1)$. Taking $i=0$, we get $\tilde{\beta}=0$, then $i=1,2$ lead to $\cos t_{0}=$ $\pm \cos \left(t_{0}+t\right)$, a contradiction.

Q.E.D.

\section{Appendix 1. Topological Vector Spaces}

We refer to [21] or [13] for topological tensor products, nuclear spaces, etc. We only fix our notations, and mention some results we need.

(A.1.1). A t.v.s. is a complex vector space $V$ with a locally convex Hausdorf vector space topology; a c.t.v.s. is a complete t.v.s.. Given t.v.s. $V_{1}$ and $V_{2}, V_{1} \simeq V_{2}$ means topological isomorphism, $V_{1} \hat{\otimes} V_{2}$ is the c.t.v.s. projective tensor product of $V_{1}$ and $V_{2}$; given t.v.s. $V_{i}, i \in I$, we endow $\prod_{i \in I} V_{i}$ with the t.v.s. product topology.

(A.1.2). We denote by $L\left(V_{1}, V_{2}\right)$ (resp: $\left.\mathscr{L}\left(V_{1}, V_{2}\right)\right)$ the space of linear maps (resp: continuous linear maps) from $V_{1}$ into $V_{2}$. There are several topologies for which $\mathscr{L}\left(V_{1}, V_{2}\right)$ is a t.v.s.: if nothing is mentioned, $\mathscr{L}\left(V_{1}, V_{2}\right)$ has the topology of uniform convergence on bounded sets. When $V_{1}$ is Montel (e.g.: $V_{1}$ quasi-complete nuclear and barreled) then $\mathscr{L}\left(V_{1}, V_{2}\right)=\mathscr{L}_{c}\left(V_{1}, V_{2}\right)$, where subscript $c$ means topology of uniform convergence on compact sets. When $V_{1}=V_{2}=V$, we use notations $L(V)$ and $\mathscr{L}(V)$ for $L(V, V)$ and $\mathscr{L}(V, V)$.

(A.1.3). We denote by $V^{*}$ the t.v.s. $\mathscr{L}(V, \mathbb{C})$. When $V$ is Montel, $V$ is reflexive, i.e. the canonical mapping from $V$ into $V^{* *}$ is an isomorphism.

(A.1.4). Assume that $V_{1}$ and $V_{2}$ are c.t.v.s., that $V_{1}$ is barreled, $V_{1}^{*}$ nuclear and complete, then $\mathscr{L}\left(V_{1}, V_{2}\right)$ is complete, and $\mathscr{L}\left(V_{1}, V_{2}\right) \simeq V_{1}^{*} \hat{\otimes} V_{2}$. These assumptions on $V_{1}$ are satisfied e.g. if $V_{1}$ is nuclear and Fréchet, or if $V_{1}$ is the dual of a nuclear and Fréchet space.

(A.1.5). Let $V_{1}$ and $V_{2}$ be two Fréchet (or dual of Fréchet) spaces; assume $V_{1}$ is nuclear, then $\left(V_{1} \hat{\otimes} V_{2}\right)^{*} \simeq V_{1}^{*} \hat{\otimes} V_{2}^{*}$. 
(A.1.6). Let $V_{i}, i \in I$, and $W$ be t.v.s., then:

$$
\left(\prod_{i \in I} V_{i}\right) \hat{\otimes} W \simeq \prod_{i \in I}\left(V_{i} \hat{\otimes} W\right) .
$$

\section{Appendix 2. Natural Topology of Countable Dimensional Vector Spaces}

We assume in Appendix 2 that $V$ is a countable dimensional vector space.

(A.2.1) Definition. An increasing sequence $\left(V_{n}\right)_{n \in \mathbb{N}}$, of finite-dimensional subspaces of $V$ is a sequence of definition if $\bigcup_{n \in \mathbb{N}} V_{n}=V$.

Given a sequence of definition, we endow $V$ with the c.t.v.s. strict inductive limit topology defined by $\left(V_{n}\right)[21]$.

(A.2.2) Lemma [21]. Any linear mapping from $V$ into a t.v.s. is continuous.

As a consequence of (A.2.2), the topology defined from $\left(V_{n}\right)$ does not depend on the choice of the sequence of definition, so we set:

(A.2.3) Definition. The strict inductive limit topology defined on $V$ from any sequence of definition is called the natural topology of $V$.

(A.2.4) Proposition [21]. The natural topology of $V$ is complete, nuclear, and Montel. $V$ is reflexive.

Given a sequence of definition $\left(V_{n}\right)$, we write $V_{n}=V_{n-1} \oplus V^{n}$, and get an isomorphism $V^{*} \simeq \prod_{n \in \mathbb{N}}\left(V^{n}\right)^{*}$, if duality is defined by: $\varphi_{n} \in\left(V^{n}\right)^{*}, v_{n} \in V$, $\left\langle\prod_{n \in \mathbb{N}} \varphi_{n}, \sum_{\text {finite }} v_{n}\right\rangle=\sum_{\text {finite }}\left\langle\varphi_{n} \mid v_{n}\right\rangle$.

This proves:

(A.2.5) Proposition. $V^{*} \simeq \prod_{n \in \mathbb{N}}\left(V^{n}\right)^{*}$ is a Fréchet space.

(A.2.6) Lemma. Let $\left(V_{n}\right)$ and $\left(V_{n}^{\prime}\right)$ be sequences of f.d. spaces, and $X$ any c.t.v.s., then:

(i) $\left(\prod_{n \in \mathbb{N}} V_{n}\right) \hat{\otimes} X \simeq \prod_{n \in \mathbb{N}}\left(V_{n} \otimes X\right)$.

(ii) $\left(\prod_{n \in \mathbb{N}} V_{n}\right) \hat{\otimes}\left(\prod_{n \in \mathbb{N}} V_{n}^{\prime}\right) \simeq \prod_{n, p}\left(V_{n} \otimes V_{p}^{\prime}\right)$.

Proof. (A.2.5) is a consequence of (A.1.6), noticing that, when $W$ is a f.d. space, then $W \otimes X=\mathscr{L}\left(W^{*}, X\right)=L\left(W^{*}, X\right)=W \otimes X$.

Q.E.D.

(A.2.7) Proposition. Let $V$ and $V^{\prime}$ be two countable dimensional vector spaces, endowed with the natural topology, then:

(i) $\left(V \hat{\otimes} V^{\prime}\right)^{*} \simeq V^{*} \hat{\otimes} V^{\prime *}$.

(ii) $V \otimes V^{\prime}=V \otimes V^{\prime}$, with natural topology.

Proof.

(i) Results from (A.2.4) and (A.1.5).

(ii) From (A.1.5) and (i), $V \hat{\otimes} V^{\prime} \simeq\left(V^{*} \hat{\otimes} V^{*}\right)^{*}$, but $V^{*}=\prod_{n \in \mathbb{N}}\left(V^{n}\right)^{*}$, $V^{\prime *}=\prod_{n \in \mathbb{N}}\left(V_{n}^{\prime}\right)^{*}$, so, from (A.2.5), $V^{*} \hat{\otimes} V^{*} \simeq \prod_{n, p}\left(V^{n}\right)^{*} \otimes\left(V^{\prime p}\right)^{*}$, so $V \hat{\otimes} V^{\prime}$

$\simeq \sum_{n, p} V^{n} \otimes V^{\prime p}$ (as t.v.s.), i.e. $V \hat{\otimes} V^{\prime}=V \otimes V^{\prime}$ with natural topology. Q.E.D. 
(A.2.8) Corollary. Any bilinear mapping from $V \times V$ into a t.v.s. is continuous.

(A.2.9). Using (A.2.2), any subspace of $V$ is closed, and has a topological supplementary. By standard orthogonality arguments, any closed subspace of $V^{*}$ has a topological supplementary (namely: the orthogonal of any supplementary subspace in $V$ of the orthogonal of the given subspace of $V^{*}$ ).

(A.2.10) We develop a very simple, but fundamental example.:

Let $V=\mathbb{C}[t] ; V_{n}=\mathbb{C}[t]_{n}$ (the subspace of polynomials of degree at most $n$ ) is a sequence of definition for the natural topology of $V$. Defining duality between polynomials and formal series as usual (cf. e.g. [21]), we get $V^{*}=\mathbb{C}[[t]] . V$ and $V^{*}$ are nuclear, Montel, reflexive, and $V^{*}$ is Fréchet.

\section{Appendix 3. Deformations of t.v.s.}

Given a vector space $V$, and a commutative algebra $A, \tilde{V}=A \otimes V$ has a natural $A$-module structure. In deformation theory [10], where $A=\mathbb{C}[[t]]$, one is rather interested in $V[[t]]=\left\{\sum_{n} t^{n} v_{n}, v_{n} \in V\right\}$, which contains $\tilde{V}$, but is generally different (unless $V$ is f.d.). Usually, one introduces a closure with respect to $t$-adic topology; unfortunately, if $V$ is a t.v.s., a $t$-adic closure will no longer be a t.v.s., so one has to introduce a coarser topology on $V[[t]]$. Since $V[[t]]=L(\mathbb{C}[t], V)=$ $\mathscr{L}(\mathbb{C}[t], V)$ (A.2.2), we define:

(A.3.1) Definition. Given a t.v.s. $V$, the associated formal space is the t.v.s. $V[[t]]=\mathscr{L}(\mathbb{C}[t], \mathrm{V})$.

The topology on $V[[t]]$ is exactly the product topology $\prod_{n} t^{n} V$, with $t^{n} V \simeq V$. It results that $\lim _{n \rightarrow \infty} t^{n} \tilde{v}_{n}=0$, for any sequence $\left(\tilde{v}_{n}\right)$ of elements of $V[[t]]$. If $V$ is complete, then $V[[t]] \simeq \mathbb{C}[[t]] \hat{\otimes} V($ A.1.4); generally if $\bar{V}$ is the completion of $V, V[[t]]$ is a dense subspace of $\bar{V}[[t]]=\mathbb{C}[[t]] \hat{\otimes} \bar{V}=\mathbb{C}[[t]] \hat{\otimes} V((\mathrm{~A} .1 .4)$ and $\mathbb{C} \hat{\otimes} V=\bar{V})$.

Given $f \in \mathbb{C}[[t]]$, and $\tilde{v} \in V[[t]]=\mathscr{L}(\mathbb{C}[t], V)$, we define $f \cdot \tilde{v}$ by $(f \cdot \tilde{v})(P)=$ $f(P) \tilde{v}(P), P \in \mathbb{C}[t]$, using $\mathbb{C}[t]^{*}=\mathbb{C}[[t]]$ (A.2.9).

Therefore $V[[t]]$ is a $\mathbb{C}[[t]]$-module; if $f=\sum_{n} f_{n} t^{n}, \tilde{v}=\sum_{n} t^{n} v_{n}$, one has:

$$
f \cdot \tilde{v}=\sum_{n} t^{n} \sum_{i+j=n} f_{i} v_{j}, \quad \text { so the map }(f, \tilde{v}) \rightarrow f \cdot \tilde{v} \text { is continuous . }
$$

(A.3.2) Definition. A topologically free (t.f.) $\mathbb{C}[[t]]$-module is a formal space $V[[t]]$ associated with a t.v.s. $V$, with its natural $\mathbb{C}[[t]]$-module structure.

We mention that we do not need to define general topological $\mathbb{C}[[t]]$-modules, but only free ones, since we are only interested in the topological version of deformation theory. Nevertheless it might be of interest to work in full generality, even for deformation theory. For the time being, t.f. $\mathbb{C}[[t]]$-modules are enough for our purpose.

(A.3.3). Given t.f. $\mathbb{C}[[t]]$-modules $V[[t]]$ and $W[[t]]$, we consider the space $\mathscr{L}_{t}(V[[t]], W[[t]])$ of continuous $\mathbb{C}[[t]]$-linear maps from $V[[t]]$ into $W[[t]]$.

(A.3.3.1) Lemma. $\mathscr{L}_{t}(V[[t]], W[[t]]) \simeq \mathscr{L}(V, W)[[t]]$, as t.v.s. and $\mathbb{C}[[t]]$ modules. 
Proof. $\mathscr{L}_{t}(V[[t]], W[[t]])$ has the t.v.s. structure induced by $\mathscr{L}(V[[t]], W[[t]])$. Given $F \in \mathscr{L}_{t}(V[[t]], W[[t]])$, let $F(v)=\sum_{n} t^{n} F_{n}(v), v \in V$; then $F_{n} \in \mathscr{L}(V, W)$, so $\widetilde{F}=\left.F\right|_{V} \in \mathscr{L}(V, W)[[t]]$. $F$ can be reconstructed from $\tilde{F}$ by:

$$
F\left(\sum_{n} t^{n} v_{n}\right)=\sum_{n} t^{n} \sum_{i+j=n} F_{i}\left(v_{j}\right) .
$$

So $F \rightarrow \tilde{F}$ will give the wanted isomorphism; it is not difficult to check that it is a topological one. Moreover, $\mathscr{L}_{t}(V[[t]], W[[t]])$ has a natural $\mathbb{C}[[t]]$-module structure defined by $(f \cdot F)(\tilde{v})=f \cdot(F(\tilde{v}))$, and $f \cdot F=f \cdot \tilde{F}$.

Q.E.D.

(A.3.3.2) Definition. The $\mathbb{C}[[t]]-d u a l$ of $V[[t]]$ is $V[[t]]_{t}^{*}=\mathscr{L}_{t}(V[[t]]$, $\mathbb{C}[[t]])$, Using (A.3.3.1):

(A.3.3.3) Proposition. $V[[t]]_{t}^{*} \simeq V^{*}[[t]]$.

(A.3.4). We denote by $\langle,\rangle_{t}$ the $\mathbb{C}[[t]]$-bilinear duality between $V[[t]]$ and $V[[t]]_{t}^{*}$.

Given $\phi \in \mathscr{L}_{t}(V[[t]], W[[t]]) \simeq \mathscr{L}(V, W)[[t]], \quad$ we write $\phi=\sum_{n} t^{n} \phi_{n}$, $\phi_{n} \in \mathscr{L}(V, W)$ and define the transpose ${ }^{T} \phi=\sum_{n} t^{n T} \phi_{n} \in \mathscr{L}\left(W^{*}, V^{*}\right)[[t]] \simeq$ $\mathscr{L}_{t}\left(W[[t]]_{t}^{*}, V[[t]]_{t}^{*}\right)$. One has:

$$
\left\langle\left.\tilde{v}\right|^{T} \phi\left(\tilde{w}^{*}\right)\right\rangle_{t}=\left\langle\phi(\tilde{v}) \mid \tilde{w}^{*}\right\rangle_{t} \quad \text { if } v \in V[[t]], \tilde{w}^{*} \in W[[t]]_{t}^{*}=W^{*}[[t]] .
$$

(A.3.5) We now define the notion of (topological) $\mathbb{C}[[t]]$-tensor product:

(A.3.5.1) Definition. The (topological) $\mathbb{C}[[t]]$-tensor product of $V[[t]]$ and $W[[t]]$ is

$$
V[[t]] \hat{\otimes}_{t} W[[t]]=(V \hat{\otimes} W)[[t]] .
$$

The characteristic property of the topological tensor product of t.v.s. is the factorization property of continuous bilinear mappings. Here, the same will hold, if one replaces bilinear by $\mathbb{C}[[t]]$-bilinear.

We need some notations. First we define a canonical continuous $\mathbb{C}[[t]]$ bilinear mapping $\tilde{i}: V[[t]] \times W[[t]]$ into $V[[t]] \hat{\otimes}_{t} W[[t]]$ by:

$$
\tilde{i}\left(\sum_{n} t^{n} v_{n}, \sum_{n} t^{n} w_{n}\right)=\sum_{n} t^{n} \sum_{i+j=n} v_{i} \otimes w_{j}, v_{n} \in V, w_{n} \in W .
$$

Now, it is quite natural to use the notation:

$$
\tilde{i}(\tilde{v}, \tilde{w})=\tilde{v} \otimes_{t} \tilde{w}, \quad \tilde{v} \in V[[t]], \quad \tilde{w} \in W[[t]] .
$$

(A.3.5.2) Proposition. Given a continuous $\mathbb{C}[[t]]$-bilinear map $\tilde{F}: V[[t]] \times W[[t]]$ $\vec{\sigma} X[[t]]$, where $X$ is a c.t.v.s., there exists a continuous $\mathbb{C}[[t]]$-linear map $\tilde{G}: V[[t]] \otimes_{t} W[[t]] \rightarrow X[[t]]$ such that:

$$
F(\tilde{v}, \tilde{w})=\tilde{G}\left(\tilde{v} \otimes_{t} \tilde{w}\right) .
$$


Proof. Given $v \in V, w \in W, \tilde{F}(v, w)=\sum_{n} t^{n} F_{n}(v, w), F_{n} \in \mathscr{L}_{2}(V, W ; X)$, so $F_{n}(v, w)=$ $G_{n}(v \otimes w)$ with $G_{n} \in \mathscr{L}(V \otimes W, X)$. Now

$$
\begin{aligned}
F(\tilde{v}, \tilde{w}) & =\tilde{F}\left(\sum_{n} t^{n} v_{n}, \sum_{n} t^{n} w_{n}\right)=\sum_{n} t^{n} \sum_{i+j+k=n} F_{k}\left(v_{i}, w_{j}\right) \\
& =\sum_{n} t^{n} \sum_{i+j+k=n} G_{k}\left(v_{i} \otimes w_{j}\right)=\left(\sum_{n} t^{n} G_{n}\right)\left(\sum_{n} t^{n} \sum_{i+j=n} v_{i} \otimes w_{j}\right) \\
& =\tilde{G}\left(\tilde{v} \otimes_{t} \tilde{w}\right),
\end{aligned}
$$

if one defines

$$
\begin{aligned}
\tilde{G} & =\sum_{n} t^{n} G_{n} \in \mathscr{L}(V \hat{\otimes} W, X)[[t]]=\mathscr{L}_{t}((V \hat{\otimes} W)[[t]], X[[t]]) \\
& =\mathscr{L}_{t}\left(V[[t]] \hat{\otimes}_{t} W[[t]], X[[t]]\right) .
\end{aligned}
$$

\section{Appendix 4. Vocabulary}

There are several, more or less restrictive, notions of algebras, bialgebras, Hopf algebras, etc. in the literature, so we give precise definitions of the notions we use in this paper, including corresponding topological definitions.

(A.4.1). A vector space $A$ (resp: c.t.v.s.) is an algebra (resp: a topological algebra) if one has fixed a linear (resp: continuous linear) map $\mu: A \otimes A$ (resp: $A \hat{\otimes} A$ ) $\rightarrow A$. As usual, we note $\mu\left(a \otimes a^{\prime}\right)=a a^{\prime}, a, a^{\prime} \in A$.

(A.4.2). An associative (resp: topological associative) algebra is an algebra (resp: topological algebra) with an associative product and a unit element.

(A.4.3). When $A$ is an algebra (resp: a topological algebra) then $A \otimes A$ (resp: $A \hat{\otimes} A$ ) is also an algebra (resp: a topological algebra), the product being defined by:

$$
(a \otimes b) \cdot\left(a^{\prime} \otimes b^{\prime}\right)=\left(a a^{\prime}\right) \otimes\left(b b^{\prime}\right), \quad a, a^{\prime}, b, b^{\prime} \in A .
$$

(A.4.4). A bialgebra (resp: a topological bialgebra) is an algebra (resp.: a topological algebra) with a morphism (resp: continuous morphism) $\Delta: A \rightarrow A \otimes A$ (resp: $A \hat{\otimes} A$ ).

(A.4.5). Hopf algebras are defined as in [6]. Topological Hopf algebras are defined by adding the continuity condition of the antipode and counit.

Acknowledgements. The authors are very happy to thank Wilfried Schmid for inspiring discussions, and Daniel Sternheimer for helpful comments and a very careful reading of the manuscript.

\section{References}

1. Bayen, F., Flato, M., Fronsdal, C., Lichnerowicz, A., Sternheimer, D.: Quantum mechanics as a deformation of classical mechanics. Lett. Math Phys. 1, 521-530 (1977); Deformation theory and quantization I and II. Ann. Phys. 111, 61-110, 111-151 (1978)

2. Bonneau, P.: Cohomology and associated deformations for non-necessarily coassociative bialgebras. Lett. Math. Phys. 26, 277-280 (1992) 
3. Bonneau, P., Flato, M., Pinczon, G.: A natural and rigid model of quantum groups. Lett. Math. Phys. 25, 75-84 (1992)

4. Chevalley, C.: Theory of Lie groups. Princeton, NJ: Princeton University Press, 1946

5. De Concini, C., Kac, V.G.: Representations of quantum groups at roots of 1 . Colloque Dixmier 1990, pp. 471-506; Progress in Math. 92, Birkhäuser

6. Drinfeld, V.G.: Quantum groups. A.M. Gleason (ed.) Proc. ICM 1986, AMS, Providence (1987), Vol. 1, pp. 798-820

7. Drinfeld, V.G.: Quasi-Hopf algebras. Leningrad Math. J. 1, 1419-1457 (1990)

8. Drinfeld, V.G.: On almost cocommutative Hopf algebras. Leningrad Math. J. 1, 321-342 (1990)

9. Faddeev, L.D., Reshetikhin, N.Y., Takhtajan, L.A.: Quantization of Lie groups and Lie algebras. Leningrad Math. J. 1, 193-226 (1990)

10. Gerstenhaber, M.: On the deformations of rings and algebras. Ann. Math. 79, 59-103 (1964)

11. Gerstenhaber, M., Giaquinto, A., Schack, S.D.: Quantum symmetry. In: Lect. Notes in Math. 1510 (1991), Berlin, Heidelberg, New York: Springer pp. 9-46

12. Gerstenhaber, M., Schack, S.D.: Algebras, bialgebras, quantum groups and algebraic deformations Contemp. Math. 134, 51-92 (1992)

13. Grothendieck, A.: Produits tensoriels topologiques et espaces nucléaires. Mem. Am. Math. Soc. 16 (1955)

14. Guichardet, A.: Cohomologie des groupes topologiques et des algèbres de Lie. Cedic/Paris: F. Nathan 1980

15. Helgason, S.: Differential geometry and symmetric spaces. New York: Academic Press, 1962

16. Jimbo, M.: A $q$-difference analogue of $\mathscr{U}(g)$ and the Yang-Baxter equation. Lett. Math. Phys. 10, 63-69 (1985)

17. Lesimple, M., Pinczon, G.: Deformations of representations of Lie groups and Lie algebras. J. Math. Phys., 34, 4251-4272 (1993)

18. Pinczon, G., Simon, J.: Extensions of representations and cohomology. Rep. Math. Phys. $161,49-77$ (1979)

19. Reshetikhin, N.: Multiparameter Quantum groups and twisted quasi-triangular Hopf algebras. Lett. Math. Phys. 20, 331-335 (1990)

20. Samelson, H.: Notes on Lie algebras. Amsterdam: Van Nostrand Reinhold (1969)

21. Trèves, F.: Topological vector spaces, distributions and kernels. New York, London: Academic Press 1967

22. Truini P., Varadarajan V.S.: Quantization of reductive Lie algebras: construction and universality. Rev. Math. Phys. 5, 363-415 (1993)

23. Woronowicz, S.L.: Compact matrix pseudogroups. Commun. Math. Phys. 111, 613-665 (1987)

Communicated by A. Connes 